\title{
Morphological instability of pores and tubules
}

\author{
D. J. KIRILL \\ Institute for Mathematics and its Applications, University of Minnesota, Minneapolis, MN \\ 55455, USA \\ S. H. DAVIS AND M. J. Miksis \\ Engineering Sciences and Applied Mathematics Department, Northwestern University, \\ Evanston, IL 60208, USA \\ AND \\ P. W. VOORHEES ${ }^{\dagger}$ \\ Materials Science and Engineering Department, Northwestern University, Evanston, IL 60208, \\ USA
}

[Received 29 August 2001 and in revised form 24 May 2002]

\begin{abstract}
We present a linear stability analysis of a uniaxially stressed, hollow cylindrical tubule, where the mass transport mechanism is surface diffusion driven by surface curvature- and elastic-energy. We find that there are always two distinct eigenmodes for any choice of wavenumbers, applied stress, and geometry. We also find that applied stress has a destabilizing effect, increasing the range of unstable wavenumbers. For any choice of applied stress and geometry, the most dangerous mode is axisymmetric, and can be either sinuous or varicose depending on choices of geometry and applied stress. The case of a cylindrical pore in a stressed infinite solid emerges as a limiting case.
\end{abstract}

Keywords: Interfaces; morphological stability; surface diffusion

\section{Introduction}

Thin solid films are becoming increasingly important in technological applications, especially in the rapidly expanding field of microelectronics. A thin film formed on a substrate, for instance, may evolve over time and break up into small islands, a particularly undesirable result in the case of interconnects in an integrated circuit. Mass transfer may occur by a number of mechanisms, for example evaporation/condensation, chemical dissolution, electromigration, and bulk, and surface diffusion; the one typically dominant at elevated temperatures is surface diffusion. The seminal work on evolution by surface diffusion, Mullins [11], formulates the equations for surface energy-driven diffusion. This model has been extended to more complicated situations, for example rods and spheres [12], lines on substrates [10], and films [15, 17, 18]. Work has also been done on extending this model to include diffusion driven by elastic-strain energy $[2,5,6,16]$.

The case of a thin, solid cylindrical rod, a whisker, evolving via capillarity-driven surface diffusion was originally discussed by Nichols and Mullins [12]. In that case, the diffusion is driven by surface energy, or equivalently, the mean curvature of the surface. Linear stability theory shows that such a whisker is unstable to small-amplitude axisymmetric perturbations whose wavelengths

†Corresponding author. Email: p-voorhees@northwestrn.edu 


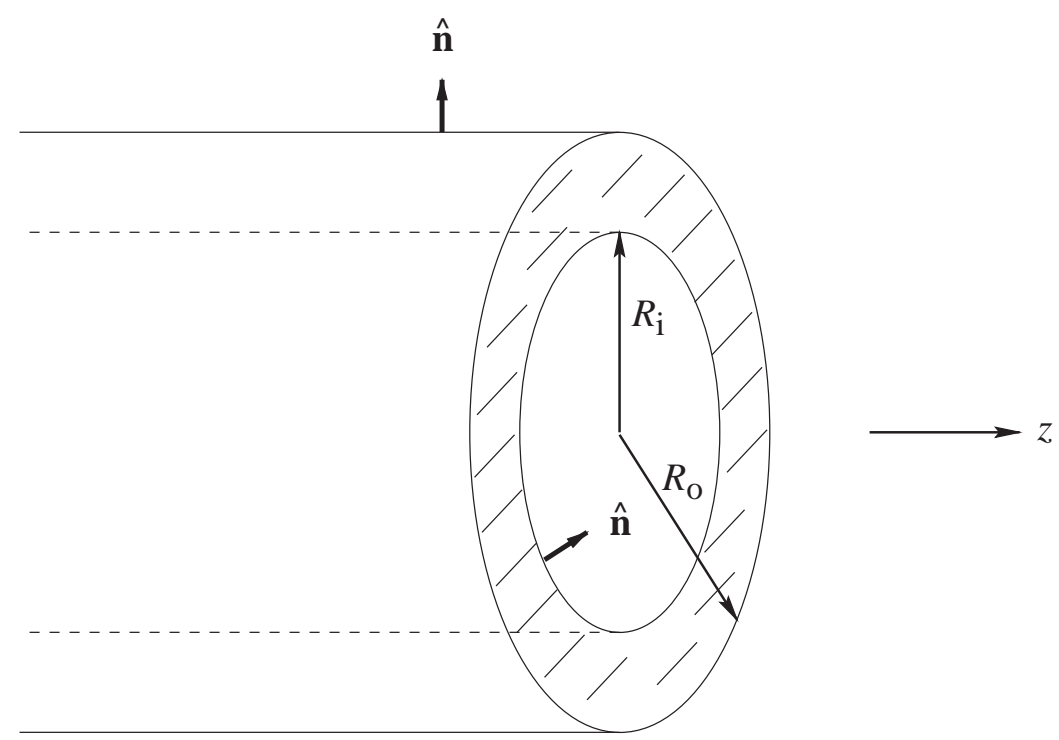

FIG. 1. The geometry of the tubule.

exceed the circumference of the unperturbed cylinder, a result identical to the classical Rayleigh instability of a liquid jet with surface tension.

The effects of elastic stress on morphological stability were studied initially by Asaro and Tiller [2], who considered the combined effects of both capillary- and stress-driven surface diffusion on the morphology of a two-dimensional semi-infinite solid under tensile loading. Their linear analysis demonstrates that the wavenumber of the fastest growing mode is proportional to the square of applied stress and inversely proportional to surface tension. Sridhar et al. [16] examined the instability of the interphase interface in a composite composed of plate and rod precipitates of one material embedded in a matrix of another. They, likewise, found that applied stress always has a destabilizing influence. Colin et al. [6] examined the case of a stressed, solid whisker subjected to axisymmetric disturbances and found that the range of unstable wavenumbers increased with increasing applied stress. They also identified maximally unstable wavenumbers in the limit of large applied stress. Kirill et al. [9] have examined capillarity- and elastic strain energy-driven surface diffusion of solid rods, with no a priori restriction on the symmetry of the perturbation. An interesting corkscrew-shaped instability was found to dominate for a range of values of applied stress. The axisymmetric case of Nicolls and Mullins is recovered as applied stress tends to zero.

This paper considers a stressed, cylindrical tubule with annular cross-section (see Fig. 1) for small-amplitude instabilities. The perturbations of the inner and outer surfaces of the tubule are coupled via the elastic fields in the material. We find that, for any choice of wavenumbers and control parameters, there are always two distinct eigenmodes, each of which represents a combined disturbance of the inner and outer surfaces. It is found that the 'most dangerous' (i.e. fastestgrowing) mode is axisymmetric for any choice of radius ratio $\eta$ and applied stress. Here $\eta=R_{\mathrm{i}} / R_{\mathrm{o}}$, where $R_{\mathrm{i}}$ and $R_{\mathrm{O}}$ are the unperturbed inner and outer radii, respectively. As $\eta \rightarrow 0^{+}$(pinhole bore), one mode takes on the characteristics of the solid whisker mode [9] (this one will be referred to as 
the solid-like mode ' $\mathrm{S}$ '), and the other begins to resemble the mode associated with the void problem (the void-like mode ' $\mathrm{V}$ '). These statements will be made more precise in the analysis that follows.

We also examine several other limiting situations, in particular the limit of small applied stress, the limit of large axial wavenumber $k$, and the 'thin-shell limit' $\left(\eta \rightarrow 1^{-}\right)$. In particular, for large $k$, a skin-depth effect is found: disturbances decay rapidly with depth into the shell. In the thin-shell limit, results compare favorably with the lamellar stability results of Sridhar et al. [16], when the inner and outer radii are made large. In addition, asymptotic expansions in 'thinness' of the shell are employed to give simple formulae for the growth rates and eigensolutions.

After formulating governing equations and boundary conditions, we analyze the stability of the 'void', a long cylindrical hole in an infinite solid matrix. The morphological instability of such microscopic channels has been observed in practice [13]: such voids have been artificially introduced in titanium ion-implanted sapphire substrates, and were seen to 'heal' during hightemperature annealing. The results presented here are related to earlier work by Colin et al. [5] in which the axisymmetric perturbations of a pore channel were studied. They analyzed the surface evolution of a cylindrical pore, when the surrounding solid is subjected to constant uniaxial stress and the perturbations are axisymmetric. Their findings for critical and most dangerous wavelengths in the large stress limit are identical to those for the whisker, as they should be; in either case, the cutoff and most dangerous wavelengths are small (when scaled on pore/whisker radius), and a curved surface appears flat when seen on a small length scale. In other words, they recover the values obtained by Grinfel'd [7] for a stressed planar surface. In addition, it is found that there is a qualitative difference between the \pm 1 modes between the void and the solid whisker; as $k \rightarrow 0$ growth rates are bounded away from zero for the solid whisker [9] in contrast to neutrally stable modes for the void.

\section{Problem formulation}

\subsection{Equations and boundary conditions}

Consider a tubule consisting of a linearly elastic, isotropic material with traction-free boundaries. Denote these surfaces by $r=r^{\mathrm{i}}(\theta, z, t)$ and by $r=r^{\mathrm{o}}(\theta, z, t)$, respectively, where $(r, \theta, z)$ are cylindrical coordinates. Hence, the displacement field, $\mathbf{u}=(u, v, w)$, is governed by Navier's equations,

$$
\mathbf{0}=\nabla^{2} \mathbf{u}+\frac{1}{1-2 v} \nabla(\nabla \cdot \mathbf{u}),
$$

for $r^{\mathrm{i}}<r<r^{\mathrm{o}},|z|<\infty$, and $0 \leqslant \theta<2 \pi$. Traction-free boundary conditions on the inner and outer surfaces are

$$
\left.\mathrm{T} \cdot \hat{\mathbf{n}}\right|_{\text {surface }}=\mathbf{0},
$$

where $\mathrm{T}$ is the stress tensor and $\hat{\mathbf{n}}$ is the outward-pointing unit normal vector. We assume periodicity in the $z$-direction. The mass transport mechanism is assumed to be capillary- and elastic-energydriven surface diffusion; hence, the evolution equation relating normal-velocity of each surface to gradients in surface chemical potential is

$$
V_{\mathrm{n}}=\hat{\mathbf{n}} \cdot \frac{\partial \mathbf{x}}{\partial t}=b \nabla_{\mathrm{s}}^{2}\left(-\kappa+\frac{1}{2 \gamma} T_{i j} \varepsilon_{i j}\right),
$$


where $\mathbf{x}$ is the position vector for a point on the solid surface, $\nabla_{\mathrm{s}}^{2}$ is the surface Laplacian, $\kappa=-\nabla_{\mathrm{S}} \cdot \hat{\mathbf{n}}$ is twice the mean curvature, $\gamma$ is isotropic surface tension (equal on the two interfaces), and $T_{i j} \varepsilon_{i j}$ is strain energy density at surface point $\mathbf{x}$. The constant $b$ contains materials parameters that characterize the tubule surfaces; $b=D_{\mathrm{s}} \gamma \Omega^{2} S_{0} / k T$, where $D_{\mathrm{s}}$ is the surface diffusivity, $\Omega$ is the atomic volume, $S_{0}$ is the number of surface atoms per unit area, and $k T$ is the thermal energy.

We introduce dimensionless variables: $\mathbf{x}=R_{\mathrm{o}} \mathbf{x}^{*}, t=R_{\mathrm{o}}^{4} t^{*} / b, T_{i j}=E \tilde{\varepsilon} T_{i j}^{*}, \varepsilon_{i j}=\tilde{\varepsilon} \varepsilon_{i j}^{*}, \mathbf{u}=$ $\tilde{\varepsilon} R_{\mathrm{O}} \mathbf{u}^{*}$, where $E$ is Young's modulus, $\tilde{\varepsilon}$ is a dimensionless scale factor, and $T_{i j}$ and $\varepsilon_{i j}$ are stress and strain components, respectively. To be more specific, here we set $\tilde{\varepsilon}=T_{z z}^{(0)} / E$, i.e. the $z z$-component of the basic-state applied uniaxial stress (see Section 2.2) divided by Young's modulus. Notice that length, time and displacement are scaled using the constant outer radius $R_{\mathrm{O}}$ of the hollow cylindrical rod. With these scalings, and dropping asterisks, Hooke's Law becomes $T_{i j}=2 \hat{\mu} \varepsilon_{i j}+\hat{\lambda} \varepsilon_{k k} \delta_{i j}$, where $\hat{\mu}=\mu / E$ and $\hat{\lambda}=\lambda / E$. The normal-velocity condition becomes

$$
V_{\mathrm{n}}=\nabla_{\mathrm{s}}^{2}\left(-\kappa+\beta T_{i j} \varepsilon_{i j}\right)
$$

where

$$
\beta=\frac{R_{\mathrm{o}} E(\tilde{\varepsilon})^{2}}{2 \gamma} .
$$

The elastic parameter $\beta$ is a ratio of a characteristic strain energy to that of the surface energy on the outer surface in the basic state. Navier's equations (1) look the same as before.

\subsection{Linearization}

Let the basic cylindrical state be given by the displacement field $\mathbf{u}^{(0)}=-v r \hat{\mathbf{r}}+z \hat{\mathbf{z}}$ (uniaxial tension), where $v$ is the Poission ratio and, $\hat{\mathbf{r}}$ and $\hat{\mathbf{z}}$ denote unit vectors. Allow a disturbance of this state by writing

$$
\begin{gathered}
\mathbf{u}(r, \theta, z, t)=\mathbf{u}^{(0)}+\delta \mathbf{u}^{(1)}(r, \theta, z, t)+O\left(\delta^{2}\right), \\
r^{\mathrm{i}}(\theta, z, t)=\eta+\delta r_{1}^{\mathrm{i}}(\theta, z, t)+O\left(\delta^{2}\right), \\
r^{\mathrm{o}}(\theta, z, t)=1+\delta r_{1}^{\mathrm{o}}(\theta, z, t)+O\left(\delta^{2}\right) .
\end{gathered}
$$

Here, $\delta$ is the amplitude of the perturbations assumed to be small in magnitude. The linear stability theory corresponds to retaining only the $O(\delta)$ terms.

Linearizing the traction-free boundary conditions yields the three equations on $r=1$,

$$
\begin{aligned}
\left(\frac{1-v}{v} u_{1, r}+u_{1}+v_{1, \theta}+w_{1, z}\right)_{r=1} & =0 \\
\left(u_{1, \theta}+v_{1, r}-v_{1}\right)_{r=1} & =0 \\
\left(u_{1, z}+w_{1, r}\right)_{r=1} & =2(1+v) r_{1, z}^{\mathrm{o}}
\end{aligned}
$$


and on $r=\eta$ :

$$
\begin{aligned}
\left(\frac{1-v}{v} u_{1, r}+\frac{1}{\eta} u_{1}+\frac{1}{\eta} v_{1, \theta}+w_{1, z}\right)_{r=\eta} & =0 \\
\left(\frac{1}{\eta} u_{1, \theta}+v_{1, r}-\frac{1}{\eta} v_{1}\right)_{r=\eta} & =0 \\
\left(u_{1, z}+w_{1, r}\right)_{r=\eta} & =2(1+v) r_{1, z}^{\mathrm{i}} .
\end{aligned}
$$

Here $u_{1}, v_{1}$, and $w_{1}$ are the $r, \theta$, and $z$ components of the linearized displacement field. The normalvelocity equation on the outer surface is,

$$
r_{1, t}^{\mathrm{o}}=-\left(\partial_{\theta}^{2}+\partial_{\theta}^{4}+2 \partial_{\theta}^{2} \partial_{z}^{2}+\partial_{z}^{2}+\partial_{z}^{4}\right) r_{1}^{\mathrm{o}}+2 \beta\left(w_{1, \theta \theta z}+w_{1, z z z}\right)_{r=1}
$$

and on the inner surface it is

$$
-r_{1, t}^{\mathrm{i}}=\left(\frac{1}{\eta^{4}} \partial_{\theta}^{2}+\frac{1}{\eta^{4}} \partial_{\theta}^{4}+\frac{2}{\eta^{2}} \partial_{\theta}^{2} \partial_{z}^{2}+\frac{1}{\eta^{2}} \partial_{z}^{2}+\partial_{z}^{4}\right) r_{1}^{\mathrm{i}}+2 \beta\left(\frac{1}{\eta^{2}} w_{1, \theta \theta z}+w_{1, z z z}\right)_{r=\eta} .
$$

Since Navier's equations are linear, the $O(\delta)$ term $\mathbf{u}^{(1)}$ also satisfies the same equations. Collectively, equations (9)-(16) are a system of partial differential equations with coefficients independent of $\theta$ and $z$; hence, normal-mode solutions can be sought, i.e.

$$
\left(\begin{array}{c}
u^{(1)} \\
v^{(1)} \\
w^{(1)} \\
r_{1}^{\mathrm{i}} \\
r_{1}^{\mathrm{o}}
\end{array}\right)=\left(\begin{array}{c}
U(r) \\
V(r) \\
W(r) \\
\tilde{r}_{\mathrm{i}} \\
\tilde{r}_{\mathrm{o}}
\end{array}\right) \mathrm{e}^{\mathrm{i}(m \theta+k z)+\sigma t}
$$

where the axial wavenumber $k$ is real, the azimuthal wavenumber $m$ is an integer, and the growth rate $\sigma$ is complex. In order to solve this problem and identify $\sigma$ as a function of $m$ and $k$, we need to solve Navier's equations for the linearized displacement field $\mathbf{u}^{(1)}$. This problem can be simplified by representing $\mathbf{u}^{(1)}$ in terms of Papkovich-Neuber potentials $\psi$ and $\phi$ [14] as follows:

$$
\mathbf{u}^{(1)}=4(1-v) \boldsymbol{\psi}-\nabla(\mathbf{x} \cdot \boldsymbol{\psi}+\phi)
$$

where

$$
\begin{aligned}
\nabla^{2} \psi & =\mathbf{0}, \\
\nabla^{2} \phi & =0 .
\end{aligned}
$$

The elastic solution for the annular interior can be written as a linear combination of six terms (involving the modified Bessel functions $I_{\mathrm{m}}$ and $K_{\mathrm{m}}$ ); hence, six unknown coefficients are introduced (here we have set the $z$-component of $\psi$ to a constant to ensure periodicity of the linearized displacement in the $z$ direction). These six, along with the two perturbation amplitudes $\tilde{r}_{\mathrm{i}}$ and $\tilde{r}_{\mathrm{o}}$, satisfy an $8 \times 8$ linear system obtained by substituting (17) into the traction-free conditions, (9)-(14), and normal-velocity conditions, (15) and (16). The characteristic equation (relating growth rate $\sigma$ to wavenumbers $m$ and $k$, and to parameters $\beta$ and $\eta$ ) is obtained by setting the determinant of the coefficient matrix equal to zero. See [8] for additional details. 


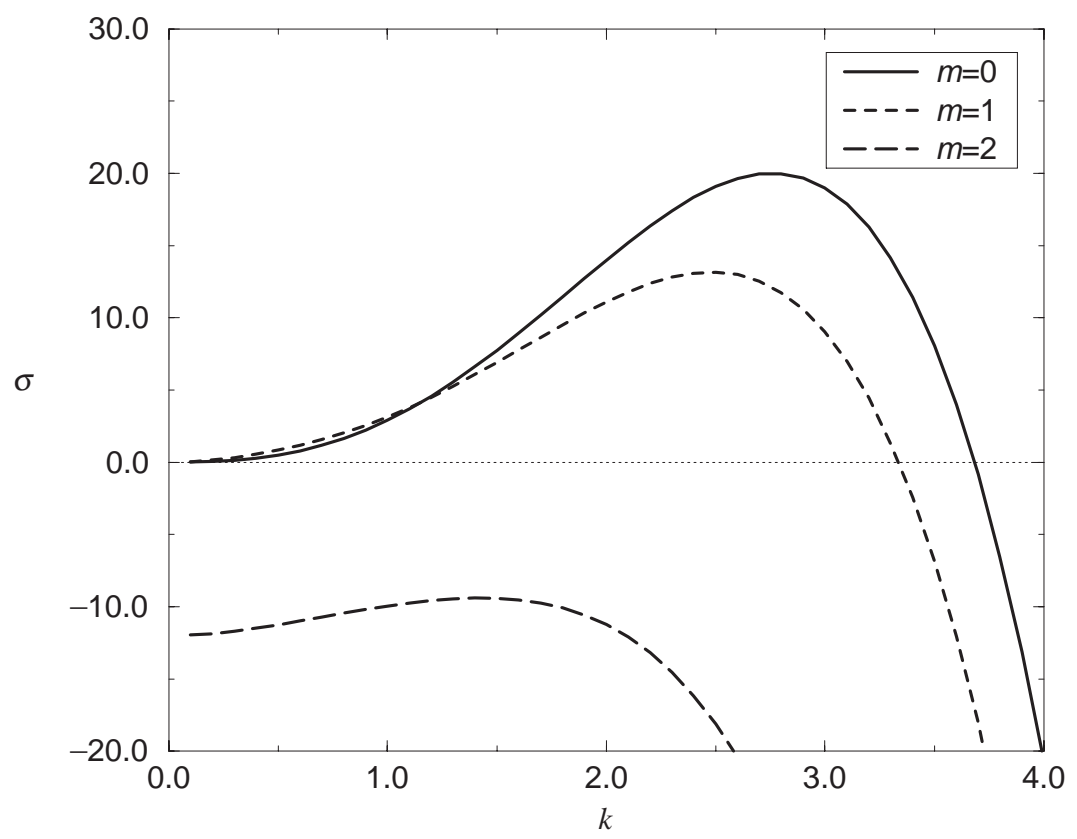

FIG. 2. Plot of growth rate $\sigma$ versus axial wavenumber $k$ for $\beta=1$ and azimuthal wavenumber $m$. Poisson ratio $v=1 / 3$.

\section{The void problem}

\subsection{Description of the problem}

Consider the special case of a cylindrical hole within a material that fills all of space. The study of pore stability is relevant to the issue of high-temperature healing of crack-like and channel-like defects in materials [13]. Given that the normal vector $\hat{\mathbf{n}}$ points out of the material, the formulation is similar to that in Kirill et al. [9], with the traction-free boundary conditions being applied on the interior surface of the cavity. In the expressions for the Papkovich-Neuber potentials, modified Bessel functions $K_{\mathrm{m}}(k r)$ (which remain bounded as $r \rightarrow \infty$ ) are required.

Figures 2 and 3 are plots of $\sigma$ versus $k$ for various values of $m$ and $\beta$. We see that the mode $m=1$ grows for $\beta=1$ and decays for $\beta=0.1$. We find that as $\beta$ increases, the number of growing azimuthal modes increases. For example, if $\beta=10$, all modes with $|m| \leqslant 15$ grow.

A typical value of $\beta$ can be estimated for a tubule within copper. From equation (5) an upper bound on $\beta$ is given by

$$
\beta=\frac{R_{\mathrm{o}}(E \tilde{\varepsilon})^{2}}{2 \gamma E} \leqslant \frac{R_{\mathrm{o}} Y^{2}}{2 \gamma E},
$$

where $Y$ is yield stress for copper. Note the inequality follows since $E \tilde{\varepsilon}=T_{z z}^{0}$ is the applied unaxial stress of the basic state and is hence bounded by $Y$. If we set $R_{\mathrm{O}}=1 \mu \mathrm{m}$, and use materials parameters $E=1.12 \cdot 10^{11} \mathrm{~Pa}, Y=6.9 \times 10^{7} \mathrm{~Pa}$, and $\gamma=1670 \mathrm{~mJ} \mathrm{~m}^{-2}[1,4]$, then $\beta \leqslant 0.013$.

For $k \rightarrow 0, \sigma^{0}$ and $\sigma^{1}$ are given approximately by (notation $\sigma^{m}$ denotes growth rate for 


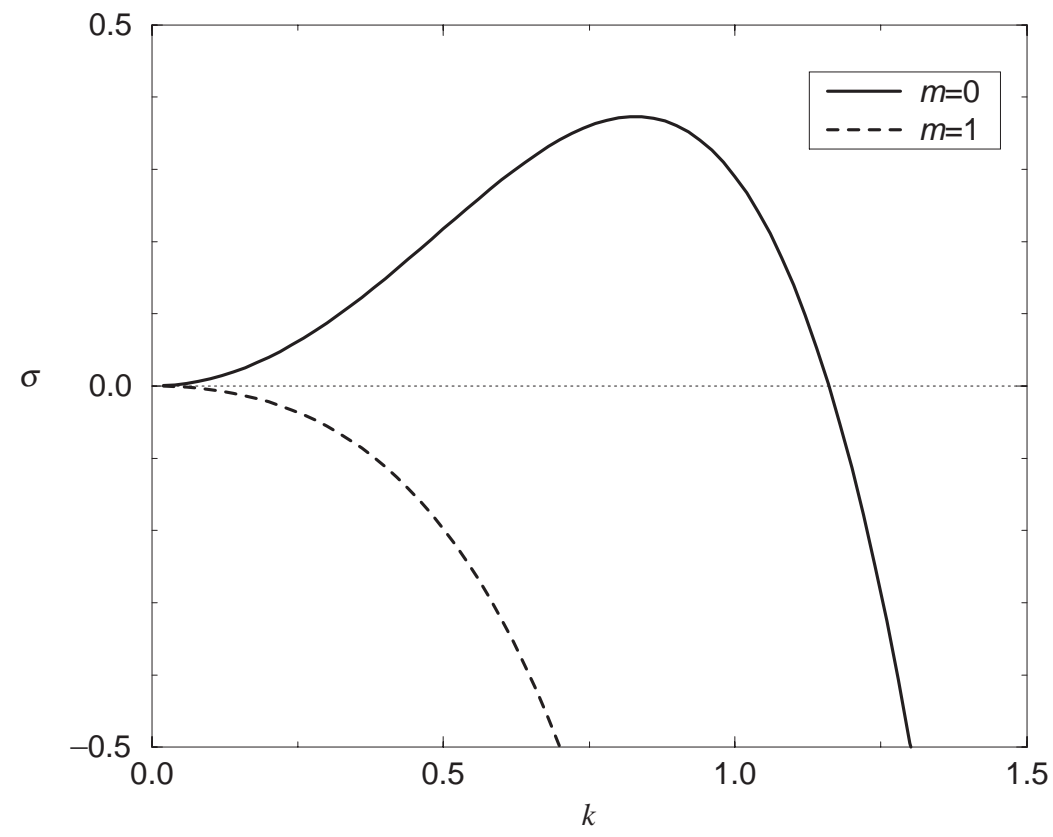

FIG. 3. Plot of growth rate $\sigma$ versus axial wavenumber $k$ for $\beta=0.1$ and azimuthal wavenumber $m$. Poisson ratio $v=1 / 3$.

azimuthal wavenumber $m$ )

$$
\begin{aligned}
& \sigma^{0}(k) \sim k^{2}-\frac{16}{3} \beta k^{4} \ln (k)+\left[-1+\beta\left(-2-\frac{16}{3} \gamma+\frac{16}{3} \ln 2\right)\right] k^{4} \\
& \sigma^{1}(k) \sim\left(-1+\frac{16}{3} \beta\right) k^{2}+\frac{35}{3} \beta k^{4} \ln (k)+\left[-1+\frac{7}{6} \beta(9+10 \gamma-10 \ln 2)\right] k^{4}
\end{aligned}
$$

$\left(\gamma \approx 0.5772\right.$ is Euler's constant) so that both $\sigma^{0}$ and $\sigma^{1}$ vanish as $k \rightarrow 0$. In contrast, for the solid whisker $\sigma^{0} \rightarrow 0$ and $\sigma^{1}>0$ as $k \rightarrow 0$ [9]. Our results imply that the dominant mode, corresponding to the largest $\sigma$ over all $k$, is always $m=0$, whereas for the whisker it is possible for modes $m= \pm 1$ to dominate.

Notice that by letting $\beta$ tend to zero in equations (22) and (23), one recovers the classical Rayleigh dispersion curves, a growing mode $m=0$ with cutoff wavenumber $k_{c}=1$, while modes with $m \geqslant 1$ decay. Recall that in the pure capillary case the instability of the cylindrical interface does not depend on whether the material lies inside or outside the cylinder.

\section{Tubules of annular cross-section}

\subsection{The dispersion relation}

Consider the hollow cylindrical rod shown in Fig. $1\left(R_{0}<\infty\right)$. There are three traction-free boundary conditions and one normal-velocity evolution equation on each surface. The characteristic equation is a quadratic in growth rate $\sigma$, so there are two distinct roots $\sigma_{1}$ and $\sigma_{2}$ for any choice of $m, k, \beta$, and $\eta$. Furthermore, these two roots are real in all ranges of $\beta$ and $\eta$ examined. 


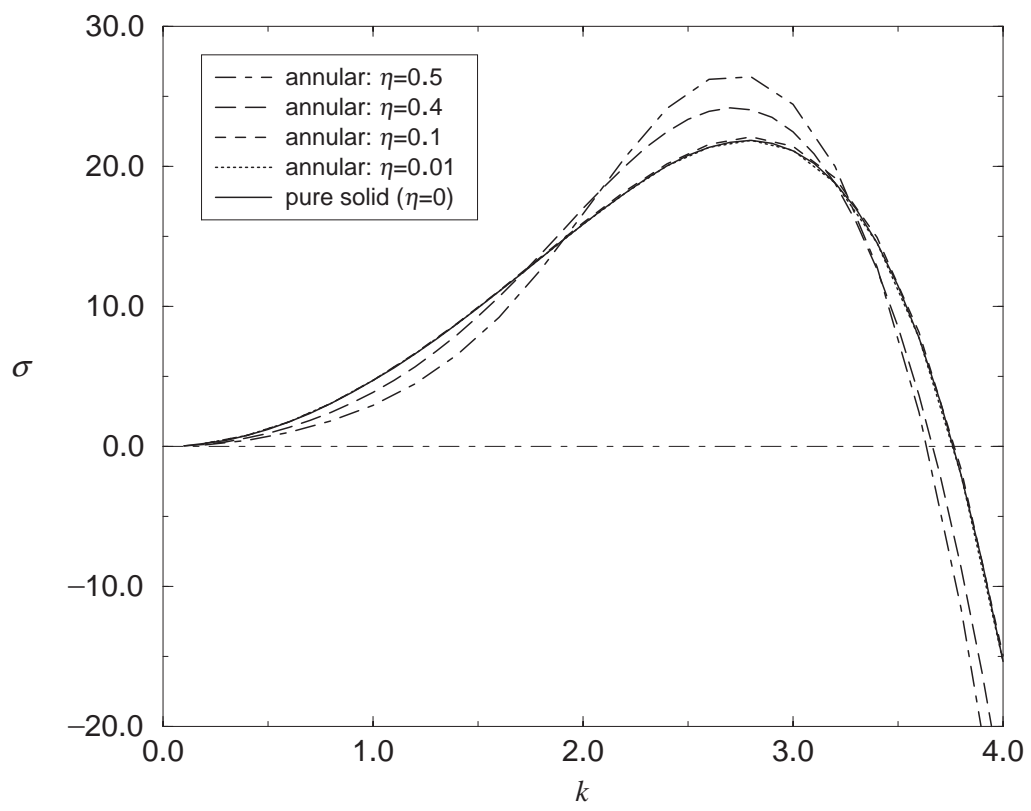

FIG. 4. Plot of growth rate $\sigma$ versus axial wavenumber $k$ for $m=0$ and $\beta=1$. Both the pure-solid case and solid-like annular mode in the limit as $\eta \rightarrow 0$ are shown. Poisson ratio is $v=1 / 3$.

As the inner radius $\eta \rightarrow 0$, one of the modes can be identified as 'solid-like' or ' $\mathrm{S}$ ', and the other as 'void-like' or ' $\mathrm{V}$ '. Graphs of $\sigma$ versus $k$ for the solid-like mode look nearly identical to those of the pure solid whisker. This is illustrated in Figs 4 and 5 for $m=0$ and $m=1$, respectively. We see that for $m=0$, independent of $\eta, \sigma$ increases from zero at $k=0$, reaches a maximum, and then decreases to negative infinity for large wavenumbers $k$. The case $m=1$ displays a similar behavior, except that $\sigma>0$ at $k=0$. If we rescale growth rate $\sigma$, wavenumber $k$, and $\beta$ using the inner radius $R_{\mathrm{i}}$ rather than outer radius $R_{\mathrm{O}}$, then the pure-void case is approximated by letting $R_{\mathrm{O}} \rightarrow \infty$ and holding $R_{\mathrm{i}}$ fixed, or equivalently taking $\eta \rightarrow 0$. We then note that dispersion curves for the void-like mode look identical to those of the pure void problem. This is illustrated in Figs 6 and 7 for the cases $m=0$ and $m=1$, respectively.

Note that the solid-like annular mode with $m=1$ (see Fig. 5) inherits the non zero growth rate at $k=0$ from the pure-solid mode. In [9], an asymptotic analysis for $k$ small shows that for a solid rod, $\sigma \sim 8 \beta$ as $k \rightarrow 0$. This predicted value for the offset is also observed here for the tubules as $\eta \rightarrow 0$. Likewise, notice in Fig. 7 that no offset is observed for any of the void-like annular modes, in agreement with the neutral stability of the pure void as $k \rightarrow 0$ (see equations (22) and (23)).

\subsection{Small inner radius limit}

Another example of the relationship between the ' $S$ ' (solid-like) mode and the $S$ (pure solid) case on the one hand, and the ' $\mathrm{V}$ ' (void-like) mode and the $\mathrm{V}$ (pure void) case can be seen in plots of $Y \equiv \tilde{r}_{\mathrm{i}} / \tilde{r}_{\mathrm{o}}$ versus $\eta$, as $\eta \rightarrow 0$. The variable $Y$ measures how the perturbation distributes itself between the inner and outer surfaces. A large $|Y|$ corresponds to most of the activity lying near the inner surface, while a small $|Y|$ implies that the activity is confined near the outer surface. Figure 8 


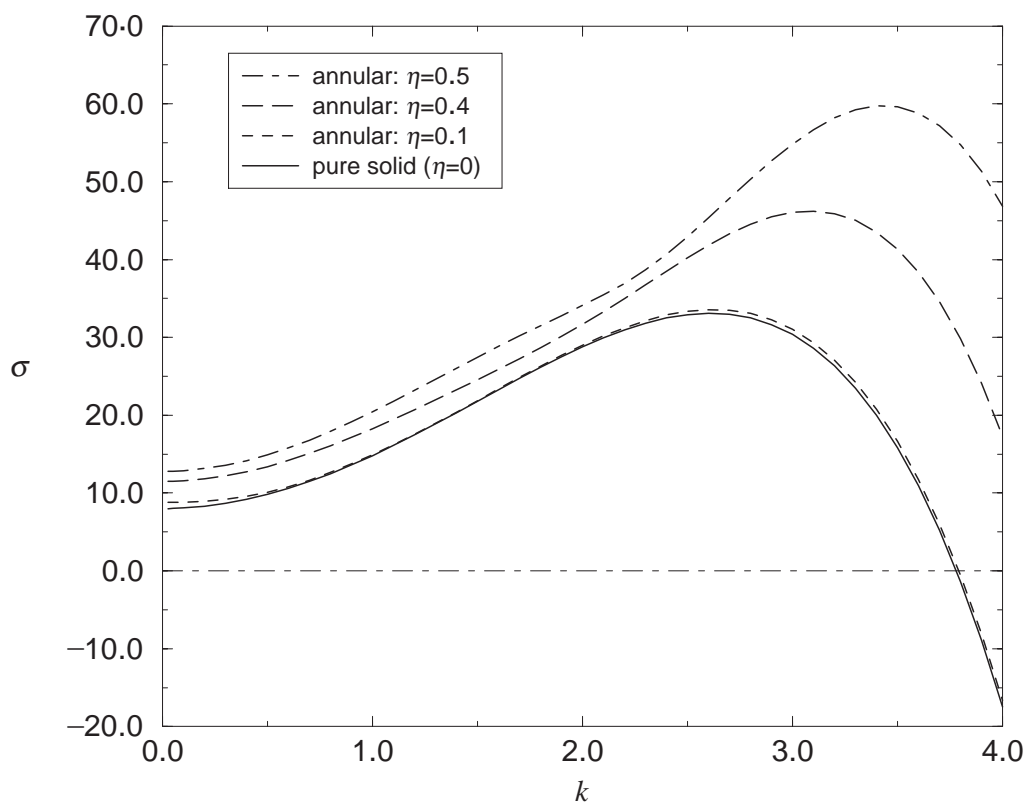

FIG. 5. Plot of growth rate $\sigma$ versus axial wavenumber $k$ for $m=1$ and $\beta=1$. Both the pure-solid case and solid-like annular mode in the limit as $\eta \rightarrow 0$ are shown. Poisson ratio is $v=1 / 3$.

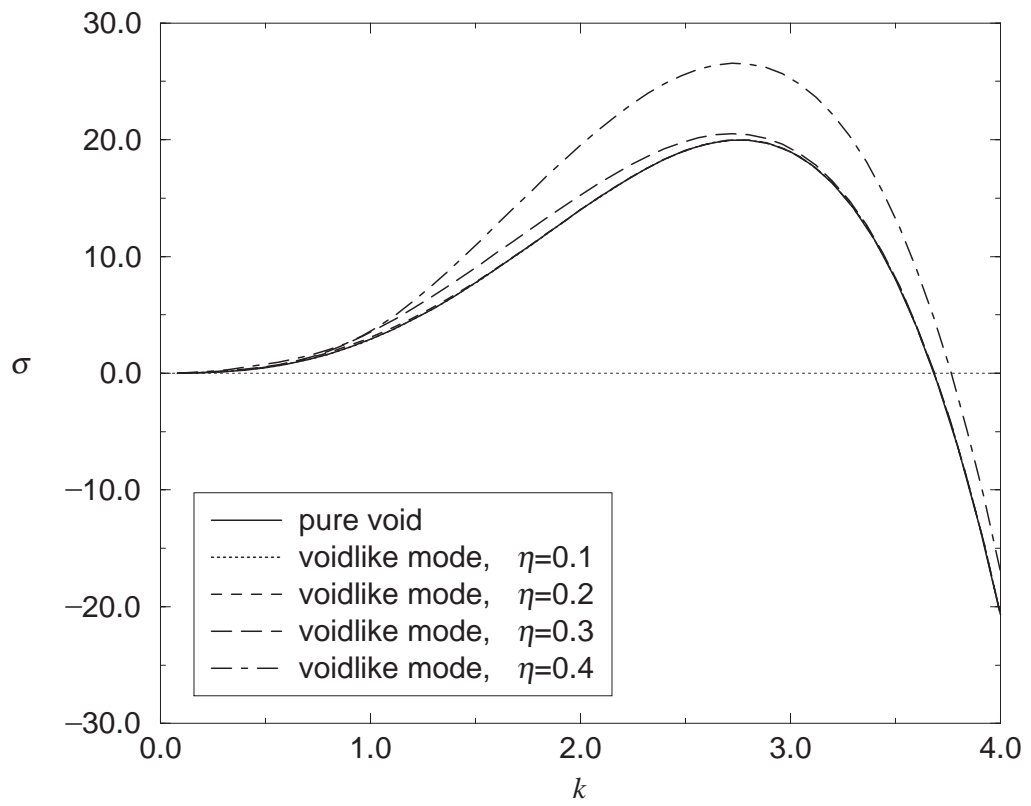

FIG. 6. Plot of $\sigma$ versus $k$ for $m=0$ and $\beta=1$ for pure-void case and void-like annular mode, in the limit as $\eta \rightarrow 0$. Note that $\sigma, k$, and $\beta$ are scaled using inner radius $R_{\mathrm{i}}$. Poisson ratio is $v=1 / 3$. 


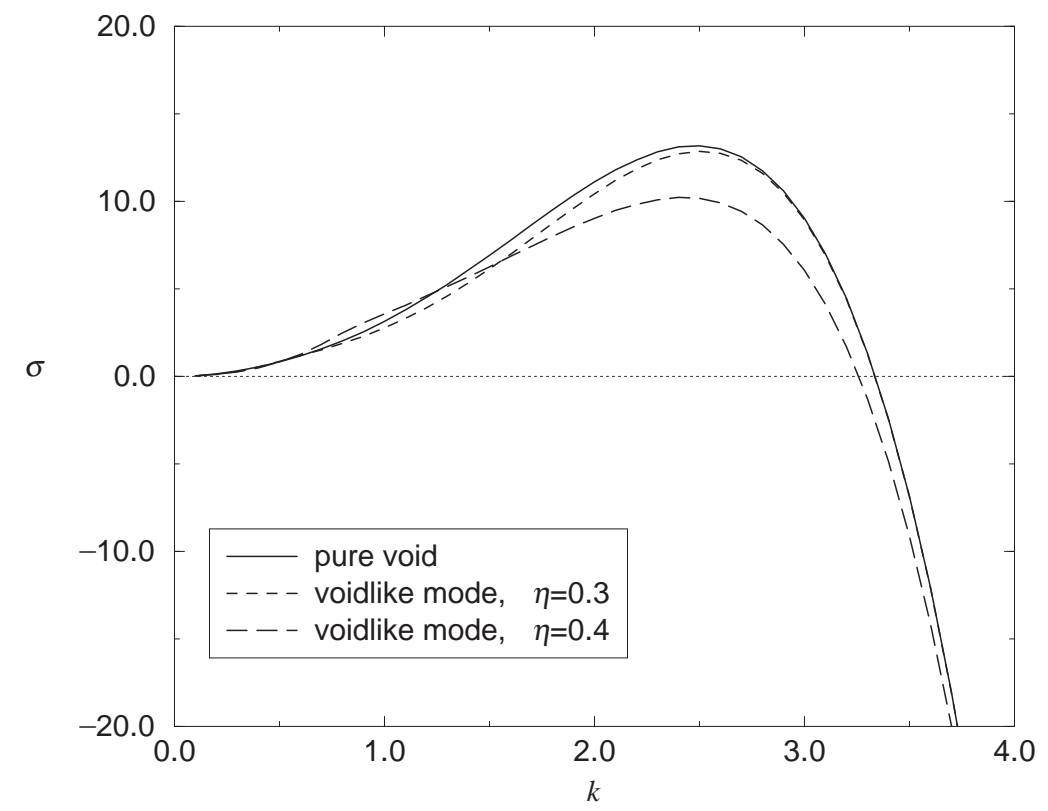

FIG. 7. Plot of $\sigma$ versus $k$ for $m=1$ and $\beta=1$ for pure-void case and void-like annular mode, in the limit as $\eta \rightarrow 0$. Note that $\sigma, k$, and $\beta$ are scaled using inner radius $R_{\mathrm{i}}$. Poisson ratio is $v=1 / 3$.

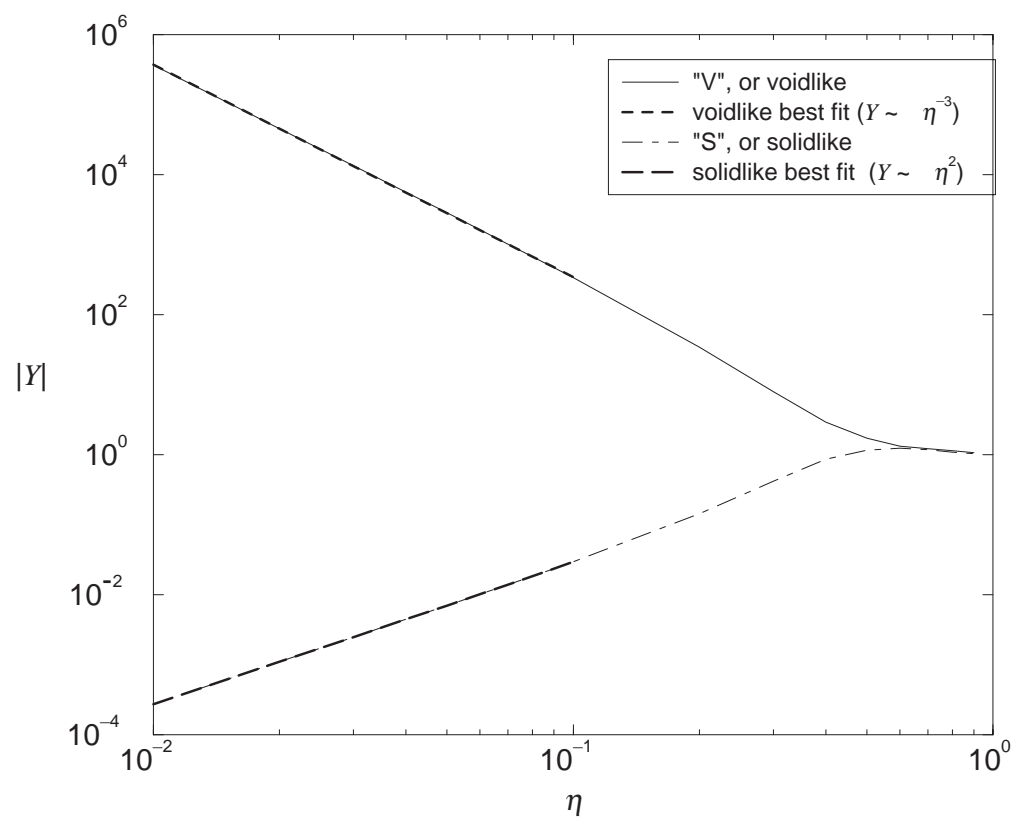

FIG. 8. Plot of $|Y| \equiv\left|\tilde{r}_{\mathrm{i}} / \tilde{r}_{\mathrm{o}}\right|$ versus $\eta$ in the limit $\eta \rightarrow 0$ for $m=0$. Also, $\beta=1, k=1$, and $v=1 / 3$. 


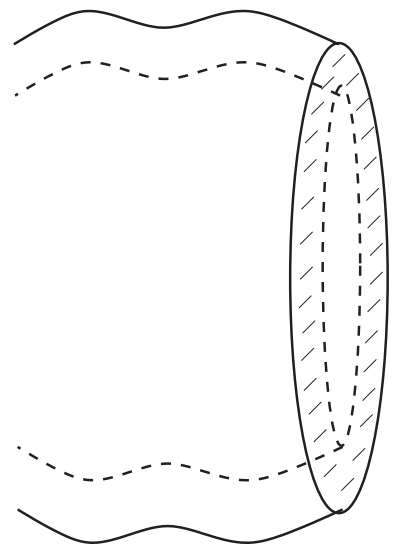

(a)

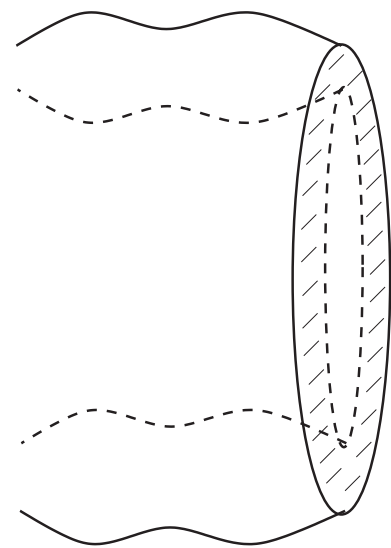

(b)

FIG. 9. (a) Sinuous mode, (b) varicose mode.

TABLE 1

Asymptotic relations for amplitude ratio as $\eta \rightarrow 0$ for the solid-like eigenmodes. Here, $\beta=1, k=1$, and Poisson ratio $v=1 / 3 . Y \equiv \tilde{r}_{\mathrm{i}} / \tilde{r}_{\mathrm{o}}$.

\begin{tabular}{ccc}
\hline$m$ & Asymptotics & Phase relationship (between $\tilde{r}_{\mathrm{i}}$ and $\tilde{r}_{\mathrm{o}}$ ) \\
\hline 0 & $Y \propto \eta^{2}$ & sinuous \\
1 & $Y \propto \eta$ & varicose \\
2 & $Y \propto \eta^{4}$ & varicose \\
\hline
\end{tabular}

TABLE 2

Asymptotic relations for amplitude ratio as $\eta \rightarrow 0$ for the void-like eigenmodes. Here, $\beta=1, k=1$, and Poisson ratio $v=1 / 3 . Y \equiv \tilde{r}_{\mathrm{i}} / \tilde{r}_{\mathrm{o}}$.

\begin{tabular}{ccc}
\hline$m$ & Asymptotics & Phase relationship (between $\tilde{r}_{\mathrm{i}}$ and $\tilde{r}_{\mathrm{o}}$ ) \\
\hline 0 & $Y \propto \eta^{-3}$ & varicose \\
1 & $Y \propto \eta^{-4}$ & sinuous \\
2 & $Y \propto \eta^{-7}$ & sinuous \\
\hline
\end{tabular}

illustrates this for $m=0, \beta=1$ and $k=1$. Notice how as $\eta \rightarrow 0$ the ' $\mathrm{S}$ ' amplitude ratio decreases to zero and the ' $\mathrm{V}$ ' ratio increases. This is consistent with the view that the ' $\mathrm{S}$ ' mode should begin to resemble the $\mathrm{S}$ mode when the geometry of the annulus begins to resemble that of the solid rod (i.e. as the hole radius shrinks to zero). In the ' $\mathrm{S}$ ' case, the amplitude of the inner radial perturbations $\tilde{r}_{\mathrm{i}}$ is a negligible fraction of the outer amplitude perturbation $\tilde{r}_{\mathrm{o}}$ for $\eta$ small. Likewise, in the ' $\mathrm{V}$ ' case for small $\eta$ the inner amplitude dominates relative to the outer amplitude. A similar result for the $m=1$ case can be found in [8].

These results are summarized in Tables 1 and 2 for the first several azimuthal wavenumbers $m$. The column labelled 'phase relationship' indicates whether the eigenmode is sinuous $(Y>0)$ or varicose $(Y<0)$; see Fig. 9. It should be mentioned that only these two phase relationships between the two surfaces have emerged in this work. 
Tubule: $Y$ versus $\beta$

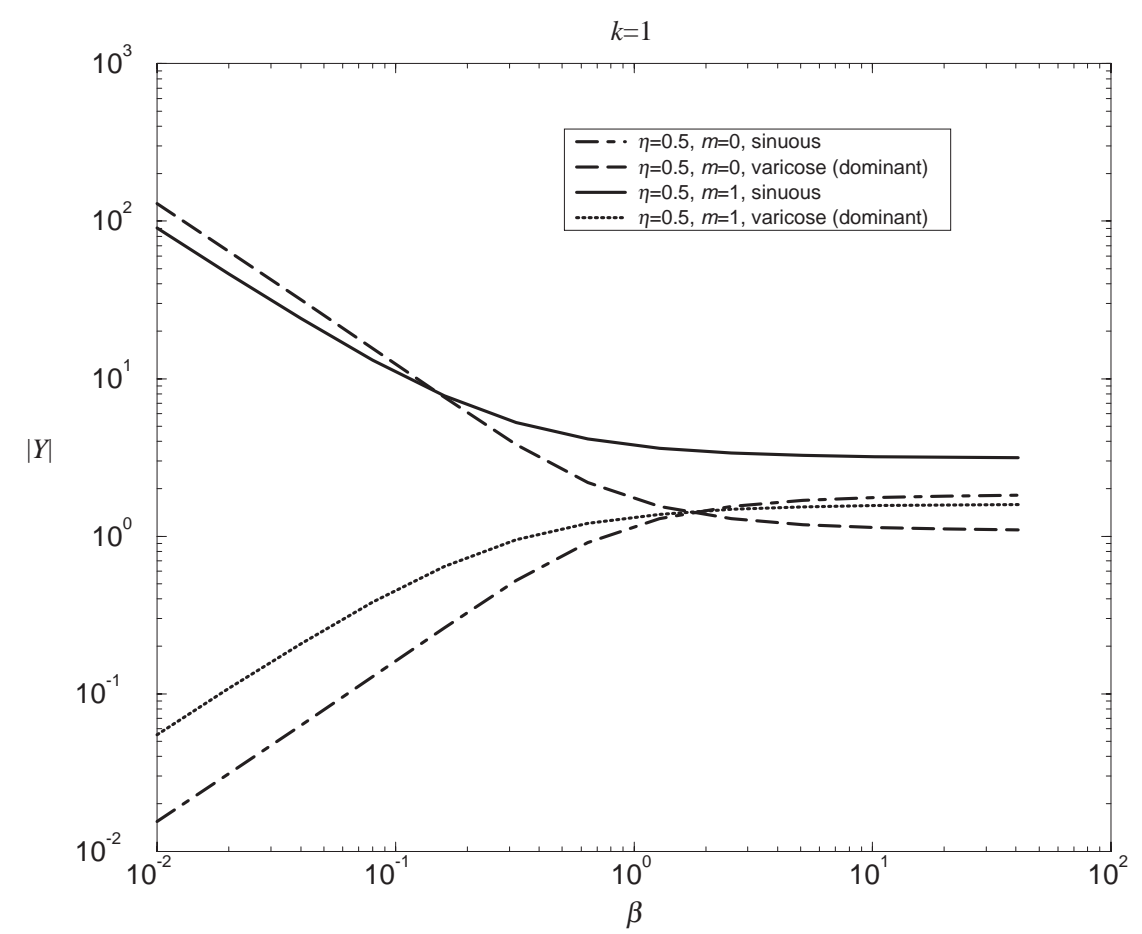

FIG. 10. Plot of $|Y|$ versus $\beta$ with fixed values of $\eta=0.5$ and $k=1$. For small values of $\beta,|Y|$ is very large for one mode, and very small for the other. The mode labelled 'dominant' is the more dangerous of the two.

\subsection{Eigenmode properties as $\beta \rightarrow 0$}

The coupling between the inner and outer surfaces of the tubule is due solely to elasticity. In the absence of applied stress $(\beta=0)$, each surface would be susceptible to only the Rayleigh instability, which is strictly a capillary effect.

A strong elastic coupling between the surfaces should result in the amplitudes of the two surface perturbations being comparable in size. One would expect that a weak coupling could result in the amplitude ratio taking on very large or very small values. These trends are clearly depicted in Fig. 10. Plotted are curves of $|Y|$ versus $\beta$ for fixed values $\eta=0.5$, and $k=1$. For both $m=0$ and $m=1$, the most dangerous mode (the 'dominant' one) happens to be varicose.

Recall that, for any choice of $\beta, \eta, m$, and $k$, there are always two modes, each with a distinct growth rate. If one denotes their amplitude ratios as $Y_{1}$ and $Y_{2}$, then our numerical results imply that the following relations appear to approximately hold independent of the value of $\beta$ :

$$
Y_{1} Y_{2}=\left\{\begin{array}{cc}
-\frac{1}{\eta}, & \text { for } m=0 \\
-\left(\frac{1}{1+k^{2}} \eta^{-3}+\frac{k^{2}}{1+k^{2}} \eta^{-1}\right), & \text { for } m=1 .
\end{array}\right.
$$

Since the product of the amplitudes is negative, then there is always one varicose and one sinuous mode. Secondly, if one ratio takes on a large value, then the second will be quite small. This explains 


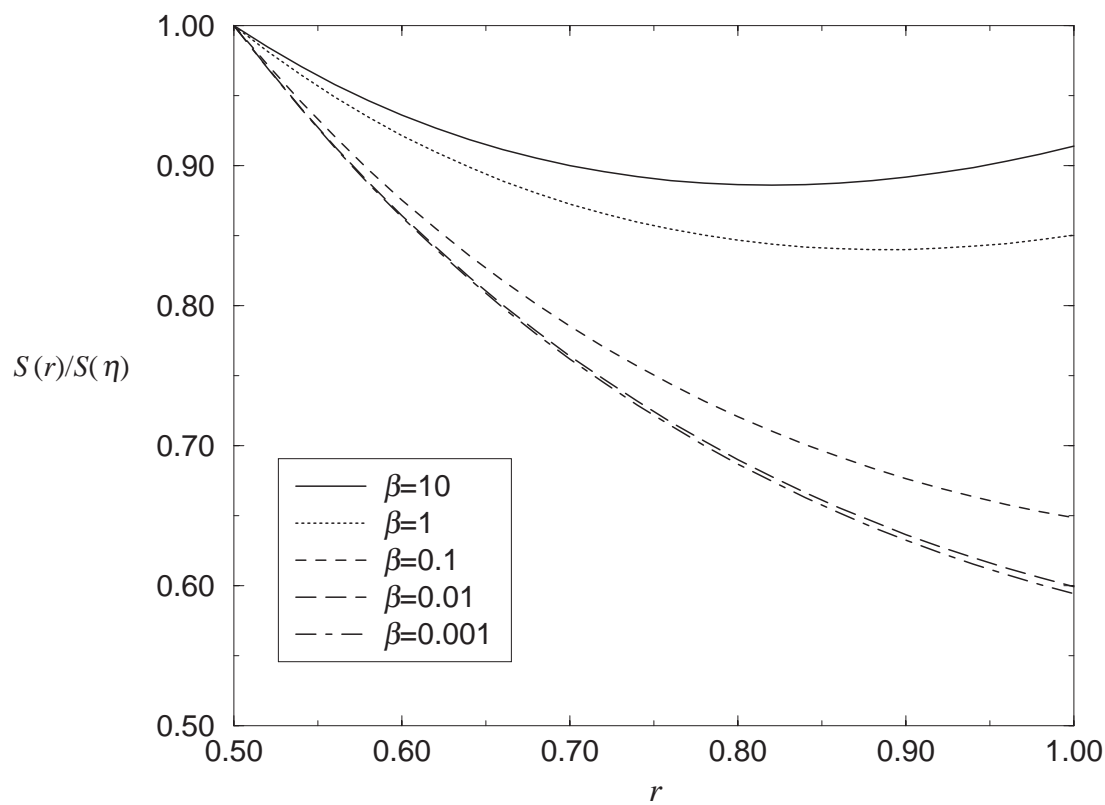

FIG. 11. Plot of elastic-strain energy perturbation (normalized to 1 at $r=\eta$ ) versus radial coordinate $r$, for the varicose mode with $\eta=0.5, m=0$ and $k=1$. Notice how the curves approach a steady shape as $\beta \rightarrow 0$.

the 'branching' of the curves in Fig. 10 as $\beta \rightarrow 0$. Note that equation (24) can only hold for non zero $\beta$ since the surface displacements will decouple for $\beta \neq 0$.

Now consider the varicose (dominant) mode with $\eta=0.5$ and $m=0$ whose amplitude ratio is depicted in Fig. 10. Since it is varicose, $Y \rightarrow-\infty$ as $\beta \rightarrow 0$. One might speculate that the $O(\delta)$ elastic strain energy term decays to zero in the neighborhood of the outer surface; this is not the case. Figure 11 shows that the normalized $O(\delta)$ strain energy density at $r=1$ approaches a steady value of approximately 0.59 as $\beta \rightarrow 0$. Therefore, the strain energy density on the outer surface remains a non zero, constant multiple of the inner-surface strain energy, even as the elastic parameter $\beta$ diminishes to zero.

\subsection{Modal properties as $k \rightarrow \infty$}

For stressed, semi-infinite slabs (e.g. films on substrate), it is well known that any surface perturbation decays to zero exponentially (in space) with characteristic length equal to the wavelength of the perturbation. This can be thought of as a skin-depth effect. A similar result is found to hold for the tubule as well. In particular, one expects that for large values of wavenumber $k$ there would be one large and one small value of $|Y|$. The large- $k$ perturbation on one surface has a correspondingly small skin depth, and so little is communicated to the other surface. Figure 12 contains a plot of $\sigma$ versus $k$ and $|Y|$ versus $k$ for $\eta=0.5, \beta=1$, and $m=0$. As $k \rightarrow \infty$, the values for $Y$ branch off to 0 and $\infty$.

An unusual phenomenon is displayed in the lower graph of Fig. 12. Near $k=2$, the two $Y$ curves exhibit poles, one blowing up and the other falling to zero at a vertical asymptote (see Kirill [8] for additional details). Furthermore, as $k$ passes through the critical value $k_{\mathrm{c}}$, the thicker curve, 

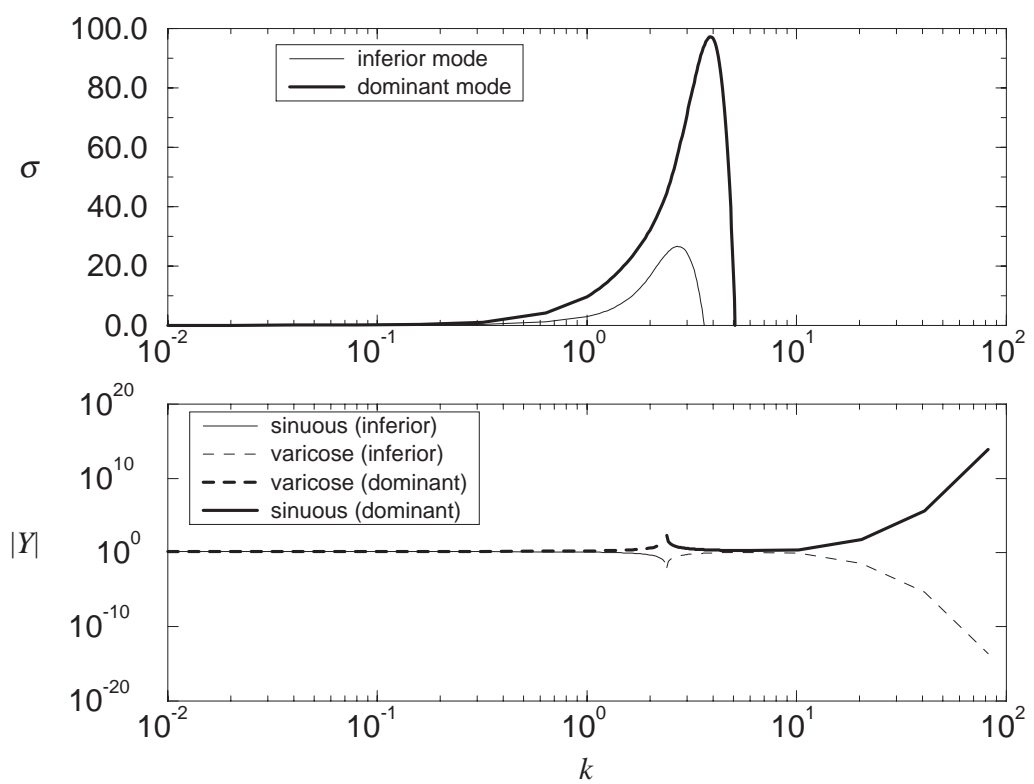

FIG. 12. Plots of growth rate $\sigma$ versus axial wavenumber $k$, and $|Y|$ vs. $k$ for $\eta=0.5, \beta=1$, and $m=0$. Notice the horizontal scale is the same for both graphs.

which corresponds to the more dangerous mode, switches from varicose to sinuous, while the other, the inferior mode, exhibits the opposite behavior. In other words, at exactly this $k_{\mathrm{c}}$, the inferior mode is composed of a nonzero (growing) perturbation on the outer surface, with a relatively quiescent inner surface. The superior mode exhibits the opposite behavior, with a perturbed inner surface and unperturbed outer surface. This is a rather counterintuitive result, considering what is known about the small- $\beta$ and large- $k$ regimes. One might think that such a behavior could only indicate the absence of elastic stress. However, the top graph of $\sigma$ versus $k$ in Fig. 12 shows, for example, that the inferior mode has a positive growth rate, indicative of stress-induced instability (a purely capillary effect would have a negative growth rate for $k>1$ ). Therefore, strain energy remains a driving force even at the pole.

As further evidence of the presence of elasticity at the pole, a plot of elastic-strain energy versus radial distance $r$ is presented in Fig. 13. More specifically, plotted on the vertical axis of Fig. 13 (bottom graph) is the $O(\delta)$ strain energy density correction $S_{1}$ (normalized to one at $r=\eta$ ), with the normal-mode prefactor $\mathrm{e}^{\mathrm{i}(m \theta+k z)+\sigma t}$ suppressed, i.e.

$$
S=S_{0}+\delta S_{1}+O\left(\delta^{2}\right)
$$

As $k$ approaches the critical value $k_{\mathrm{c}} \approx 2.39$, the strain energy on the outer surface $(r=1)$ decreases to zero, as one might suspect, and the dispersion curve is apparently unaffected as $k$ passes through the pole.

It is interesting to note that the tubule can have growing perturbations on one surface and remain unperturbed on the other with a non zero elastic parameter $\beta$. 

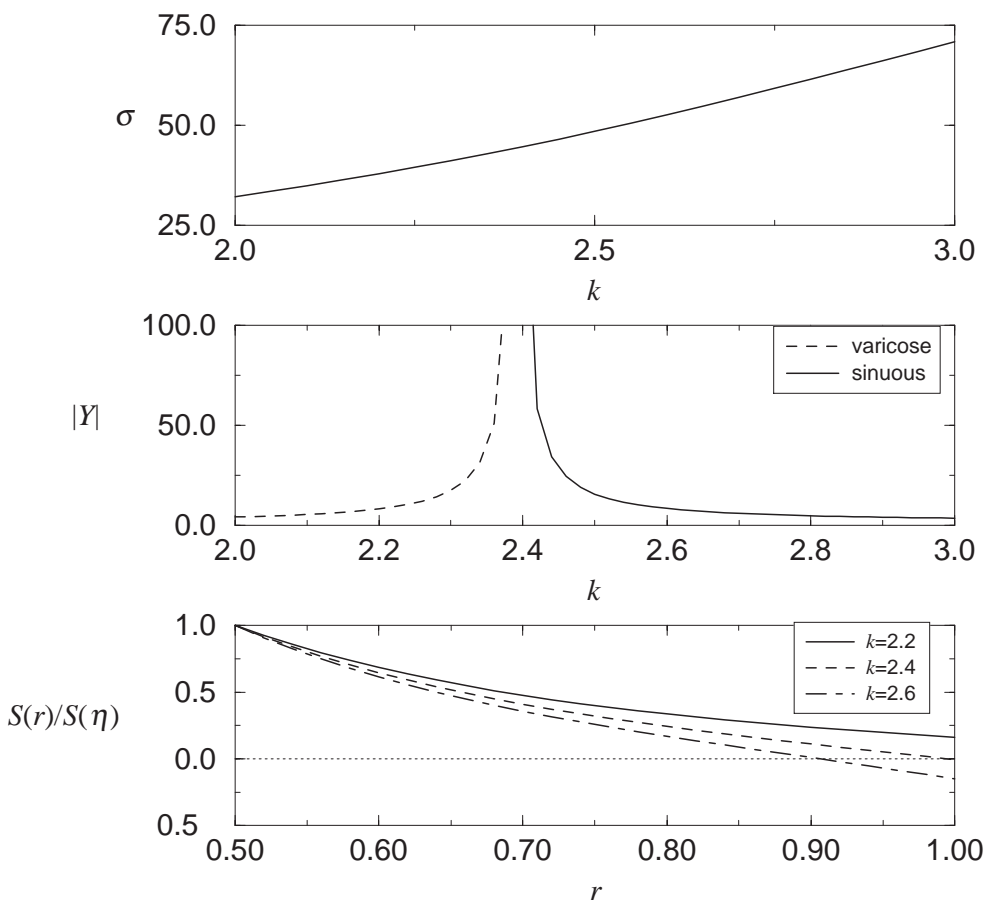

FIG. 13. The neighborhood of the pole point, see Fig. 12. Parameters are $\eta=0.5, \beta=1$, and $m=0$. Plotted are (top to bottom): growth rate $\sigma$ vs. wavenumber $k$; amplitude ratio $|Y|$ vs. $k$; normalized strain energy versus radial distance $r$. Notice that the horizontal scale for the top and middle graphs are the same.

\subsection{Plots of maximum growth rate $\sigma_{\max }$ and wavenumber $k_{\max }$ versus elastic parameter $\beta$}

Figures 14 and 15 are plots of $\sigma_{\max }$ and $k_{\max }$ versus elastic parameter $\beta$, for several values of $m$ and $\eta$. Notice that all of the curves merge as $\beta \rightarrow \infty$, independent of $m$ and $\eta$. Futhermore, they appear to merge towards a straight line, which suggests a power-law fit: i.e. for large $\beta, k_{\max } \sim \hat{k} \beta$ and $\sigma_{\max } \sim \hat{\sigma} \beta^{4}$, where $\hat{k}$ and $\hat{\sigma}$ are both $O(1)$. If we make these substitutions in the dispersion relation, and use asymptotic expansions for the modified Bessel functions $I_{\mathrm{m}}$ and $K_{\mathrm{m}}$, it is possible to show (after some algebra) that as $\beta \rightarrow \infty$,

$$
\sigma_{\max } \sim \frac{4096}{243} \beta^{4}, \quad k_{\max } \sim \frac{8}{3} \beta .
$$

These asymptotic formulae match almost identically the corresponding ones for the solid whisker; see Fig. 8 of [9].

One can demonstrate for small $\eta$ why the most dangerous modes are $m=0$ for any value of $\beta$, and this argument rests on the fact that, for small $\eta$, the dispersion curves for the ' $\mathrm{S}$ ' and ' $\mathrm{V}$ ' tubule modes are nearly identical to those of the $\mathrm{S}$ (pure solid) and V (pure vapor), respectively (see Figs 4-7). Recall that the solid whisker exhibits helical instability for $\beta$ in the range $(0.04,2.35)$ (see Section 5(a) of [9]). Further, the maximum growth rate for $\beta \approx 2.35$ is given approximately by the large- $\beta$ asymptotic formula $\sigma_{\max } \sim 16.9 \beta^{4}$; hence, we expect the maximum growth rate at the high end of the helical range to be given by $\sigma_{\max } \approx 515$. This is about the largest growth rate that the 


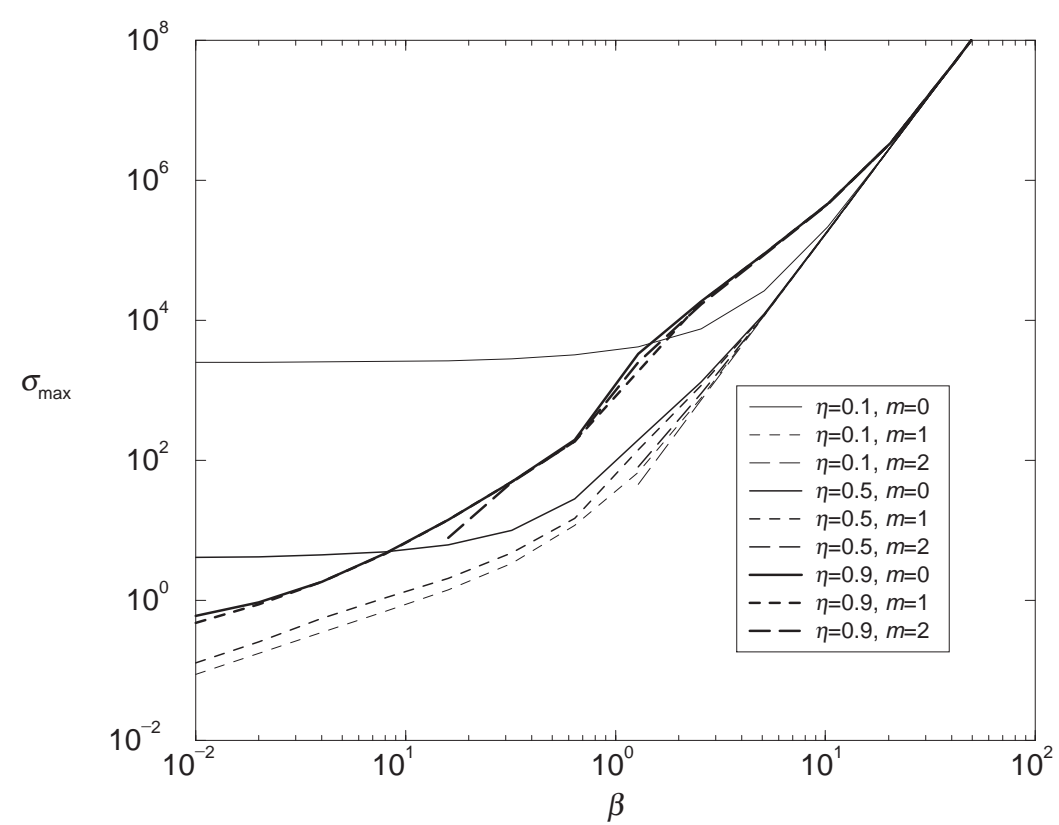

FIG. 14. Plot of maximum growth rate $\sigma_{\max }$ versus parameter $\beta$ for $m=0,1,2$ and $\eta=0.1,0.5,0.9$. Because it is a $\log -\log$ plot, negative growth rates are not shown (e.g. examine $m=2$ ). As $\beta \rightarrow \infty, \sigma_{\max } \sim 4096 \beta^{4} / 243 \approx 16.9 \beta^{4}$, independent of wavenumber $m$ and radii ratio $\eta$.

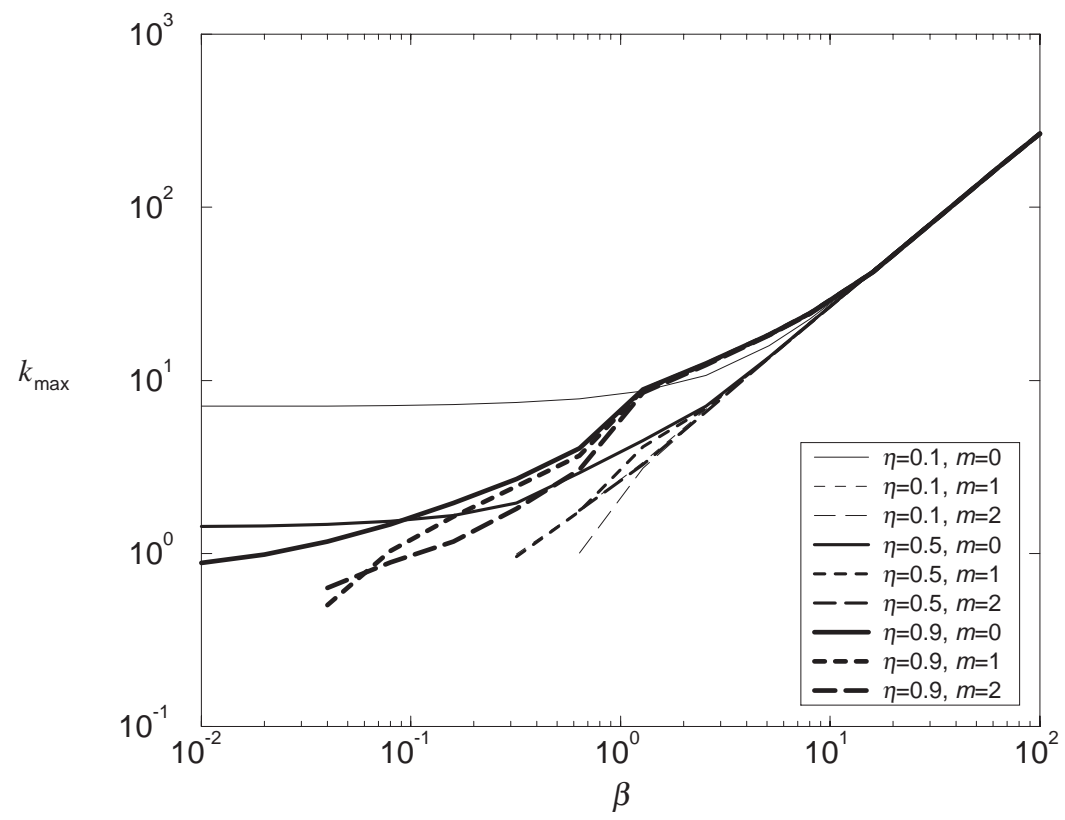

FIG. 15. Plot of maximum growth wavenumber $k_{\max }$ versus parameter $\beta$ for $m=0,1,2$. As $\beta \rightarrow \infty, k_{\max } \sim 8 \beta / 3$, independent of $m$ and $\eta$. 
solid whisker (and hence the 'S' tubule mode) could attain while exhibiting the helical instability. Note that this growth rate is based on an outer scaling: i.e. time is scaled as the fourth power of outer radius $R_{0}$. Now, let us define

$$
\beta^{\mathrm{i}}=\frac{R_{\mathrm{i}} E \tilde{\varepsilon}^{2}}{2 \gamma},
$$

the dimensionless applied stress when scaled using the inner radius. So, when $\beta=O(1)$, one has $\beta^{\mathrm{i}}=O(\eta)$, which is small. Consequently, as seen from the inner surface, the driving force for instability is predominantly capillarity, so that an axisymmetric $(m=0)$ perturbation will be excited with growth rate given by $\sigma_{\max } \approx 1 / 4$ (using inner time scaling); see Figs 2 and 3 . Now, in order to get a concrete comparison between the maximum growth rate of the ' $\mathrm{S}$ ' and ' $\mathrm{V}$ ' modes, one must first recall the relation between growth rates which are scaled on the inner radius $R_{\mathrm{i}}$ and those which are scaled on the outer radius $R_{0}: \sigma^{\text {inner }}=\eta^{4} \sigma^{\text {outer }}$. In other words, fixing $\eta=0.1$ (for illustrative purposes) and $\sigma^{\text {inner }}=\frac{1}{4}$ implies that $\sigma^{\text {outer }}=\frac{1}{4} \times 10^{4}=2500$, which is much larger than the predicted value of approximately 515 for the ' $\mathrm{S}$ ' mode. Since, the maximum growth rate for the axisymmetric void-like mode is much larger than the largest possible helical solid-like mode, then axisymmetric disturbances should dominate. Again, this argument is valid only for $\eta \ll 1$.

Notice in Figs 14 and 15 that the graphs of $\sigma_{\max }$ and $k_{\max }$ exhibit ramp-like behavior for $\eta=0.9$ in the neighborhood of $\beta=0.65$. A graphical explanation of this is presented in Figs 16 and 17, which show graphs of $\sigma_{\max },\left|Y_{\max }\right|$, and $k_{\max }$ versus $\beta$, and a series of plots of $\sigma$ versus $k$, respectively. It is clear from Fig. 17 that, for $\beta \approx 0.65$, the dominant growth curve has two distinct maxima (double hump). So, as $\beta$ passes through 0.65 , there is a jump from $k_{\max } \approx 4$ to $k_{\max } \approx 6$ : see Fig. 16. This clearly shows that, for a special choice of $\beta$, there can be two different dominant wavelengths with the same growth rate, and hence the ramp-like behavior in $\sigma_{\max }$ is present. Note that the plots in Fig. 16 contain the values for the most dangerous mode for each $\beta$ (hence, the 'max' subscripts), while the dispersion curves in Fig. 17 display both inferior and superior growth rates.

Figure 17 reveals some additional information about the varicose-to-sinuous transition. It appears that there is a critical value of $k \approx 5$, to the left of which the superior (inferior) modes are varicose (sinuous), and to right of which the superior (inferior) modes are sinuous (varicose). This critical $k$ appears to be independent of $\beta$, and is a function solely of $\eta$ (i.e. the tubule geometry). For example, a similar plot, but with $\eta=0.5$ can be found in Kirill [8]. In that case, the transition wavenumber appears to be approximately 2. These transitions occur when $Y=0$ or $Y= \pm \infty$ (i.e. a pole).

\subsection{Which mode is most favored: sinuous or varicose?}

Figure 18 reveals that, at least for $\eta=0.5$ and $\eta=0.9$, the dominant $m=0$ modes are varicose for smaller values of $\beta$, and sinuous for values of $\beta>0.5$ (very roughly). It is clear for $\eta=0.5$ that the curves blow up at the pole. For small $\eta$, namely $\eta=0.1$, the dominant $m=0$ mode is sinuous for all $\beta$ depicted in the plot. Sridhar et al. [16] state that the sinuous mode is favored over the varicose mode in the stability of lamellar composites, regardless of magnitude of applied uniaxial stress (see Section 4.7 for further discussion). As we shall see later, when a thin-shell limit $\eta \rightarrow 1^{-}$is taken, our results compare favorably with results of [16]. Results for $m=1$ which are similar to Fig. 18 and display the varicose-to-sinuous transition can be found in Kirill [8].

In fact, for large $\beta,\left|Y_{\max }\right|$ should decay exponentially with $\beta$. A rough proof of this can be 

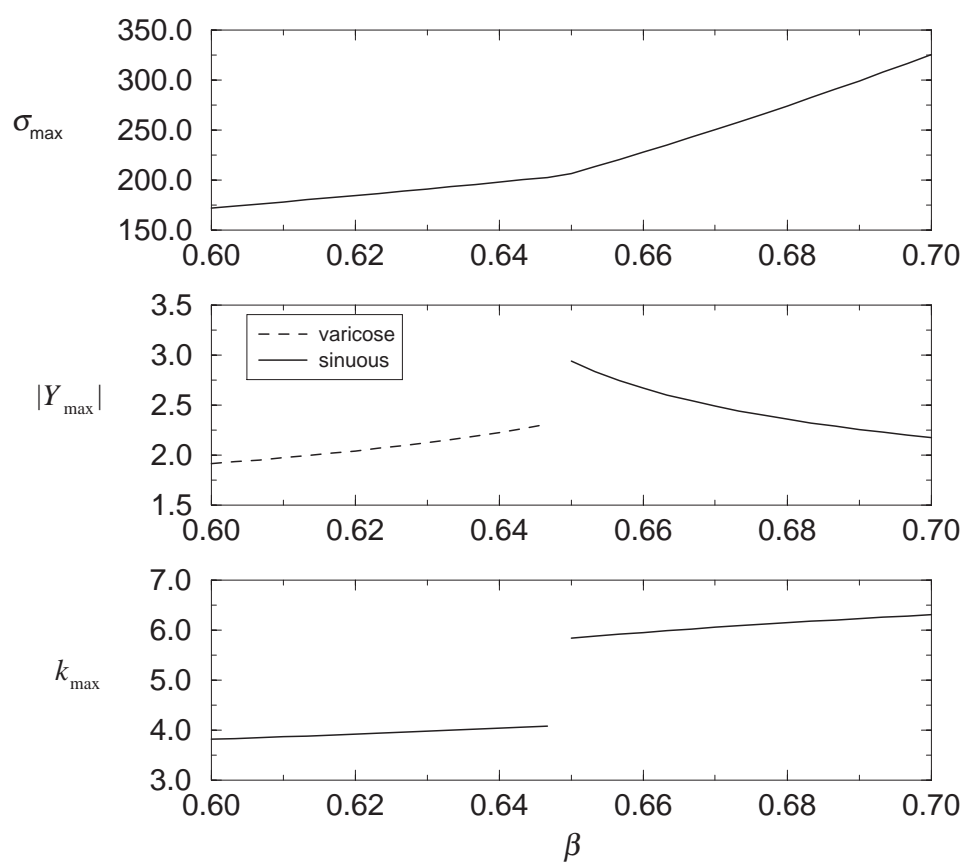

FIG. 16. Plots of $\sigma_{\max }, Y_{\max }$, and $k_{\max }$ versus $\beta$, for $\eta=0.9$ and $m=0$. The horizontal scale is the same in all three graphs.

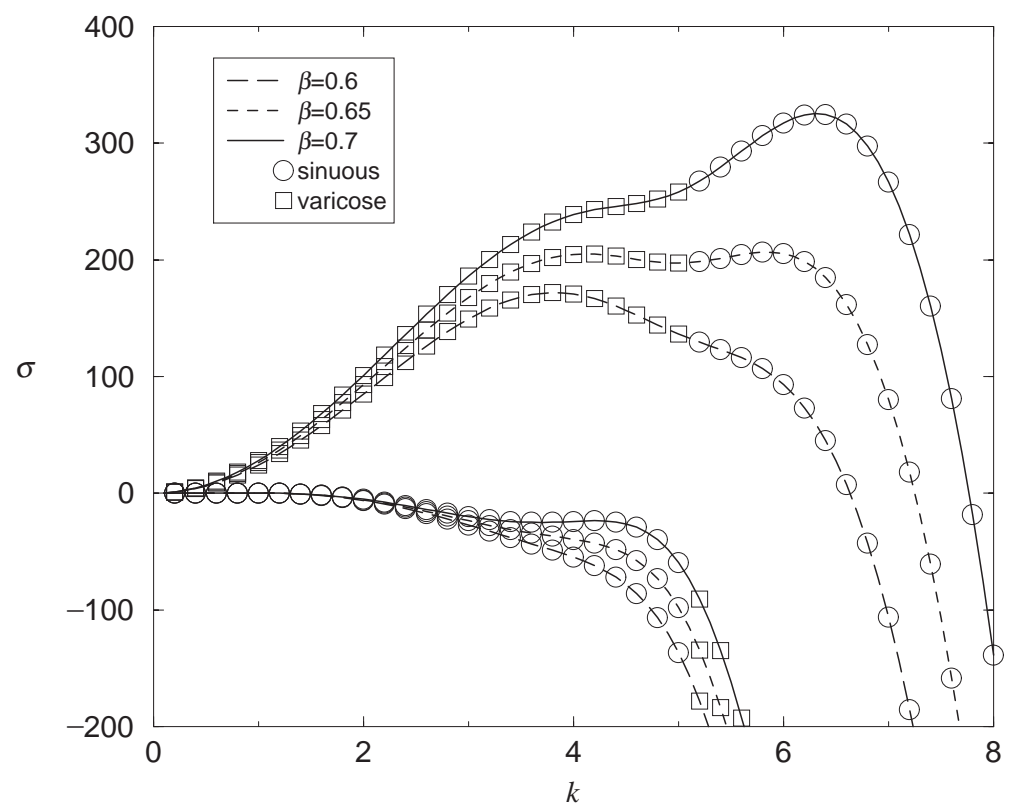

FIG. 17. A series of dispersion curves, $\sigma$ versus $k$, for various values of $\beta$, with $\eta=0.9$ and $m=0$. This complements the previous graph. 


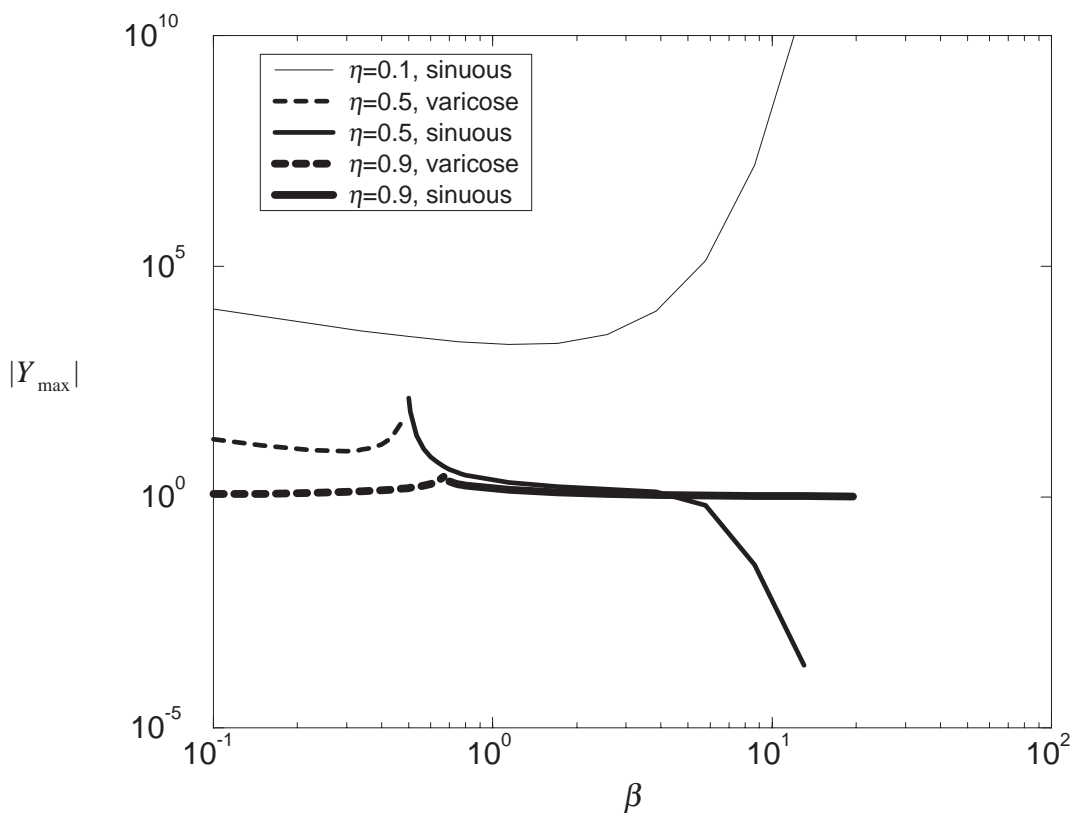

FIG. 18. Plot of $|Y| \equiv\left|\tilde{r}_{\mathrm{i}} / \tilde{r}_{\mathrm{o}}\right|$ versus $\beta$ for the most dangerous mode, with $m=0$ and $\eta=0.1,0.5,0.9$. Notice the transition from varicose to sinuous as $\beta$ increases (for $\eta=0.5$ and 0.9 ).

given. An elementary manipulation of the normal-velocity evolution equations (15) and (16) yields

$$
Y \equiv \frac{\tilde{r}_{\mathrm{i}}}{\tilde{r}_{\mathrm{o}}}=-\frac{W(\eta)}{W(1)} \frac{\sigma-k^{2}+k^{4}}{\sigma-k^{2} / \eta^{2}+k^{4}},
$$

where $W(r)$ is the radial amplitude of the linearized $z$-displacement. Recall that the displacement fields for the tubule can be written as a linear combination of modified Bessel functions $I_{\mathrm{m}}(\mathrm{kr})$ and $K_{\mathrm{m}}(k r)$. As stated in the previous section, for large $\beta$ maximum wavenumber and growth rate are given by $k_{\max } \sim 8 \beta / 3$, and $\sigma_{\max } \sim 4096 \beta^{4} / 243$, respectively. Hence large-argument asymptotic expansions for the functions $I_{\mathrm{m}}$ and $K_{\mathrm{m}}$ [3] imply that $W(r)$ will be of the form $W(r) \sim$ (constant) $\frac{e^{k_{\max } r}}{\sqrt{k_{\max } r}}$, and that equation (28) yields

$$
Y \sim(\text { constant }) \exp \left(\frac{8}{3}(\eta-1) \beta\right) .
$$

Since $\eta<1,|Y|$ decays exponentially when $\beta$ is large. Notice that when $\eta \approx 1$ this limit is non-uniform, which explains the 'flatness' of the $\eta=0.9$ curve in Fig. 18.

The fact that values of $|Y|$ are so close to unity as $\eta \rightarrow 1$ can easily be explained on the basis of symmetry. As $\eta$ approaches 1, the hollow rod looks increasingly like a thin cylindrical shell. Hence, there is a mirror symmetry present, and the magnitudes of $\tilde{r}_{\mathrm{i}}$ and $\tilde{r}_{\mathrm{o}}$ are nearly identical.

\subsection{The thin-shell limit $\left(\eta \rightarrow 1^{-}\right)$}

4.7.1 Asymptotics. Here we examine the growth rates of the sinuous and varicose eigenmodes in the limit of $\eta \rightarrow 1$. In this limit, the annulus begins to resemble a thin-shelled cylinder, with the 
inner and outer radii almost the same. Let

$$
\tilde{\eta} \equiv 1-\eta
$$

and express for $\tilde{\eta} \rightarrow 0$ Navier's equations, the traction-free boundary conditions and the normalvelocity equation. An appropriate of coordinate is

$$
\rho=1+\frac{r-1}{\tilde{\eta}},
$$

so that $\rho$ takes on values over the fixed interval $(0,1)$.

We can write the displacements and surface deflections in terms of normal modes (17). It is natural to consider asymptotic expansions as follows: for the sinuous eigenmode,

$$
\begin{aligned}
U & \sim U_{0}+\tilde{\eta} U_{1}+\cdots \\
V & \sim V_{0}+\tilde{\eta} V_{1}+\cdots \\
W & \sim \tilde{\eta}\left(W_{0}+\tilde{\eta} W_{1}+\cdots\right) \\
\tilde{r}_{\mathrm{o}} & \sim H_{0}^{\mathrm{o}}+\tilde{\eta} H_{1}^{\mathrm{o}}+\cdots \\
\tilde{r}_{\mathrm{i}} & \sim H_{0}^{\mathrm{i}}+\tilde{\eta} H_{1}^{\mathrm{i}}+\cdots \\
\sigma & \sim \sigma_{0}^{\mathrm{s}}+\tilde{\eta} \sigma_{1}^{\mathrm{s}}+\cdots
\end{aligned}
$$

and for the varicose eigenmode,

$$
\begin{aligned}
U & \sim U_{0}+\tilde{\eta} U_{1}+\cdots \\
V & \sim V_{0}+\tilde{\eta} V_{1}+\cdots \\
W & \sim W_{0}+\tilde{\eta} W_{1}+\cdots \\
\tilde{r}_{\mathrm{o}} & \sim \tilde{\eta}\left(H_{0}^{\mathrm{o}}+\tilde{\eta} H_{1}^{\mathrm{o}}+\cdots\right) \\
\tilde{r}_{\mathrm{i}} & \sim \tilde{\eta}\left(H_{0}^{\mathrm{i}}+\tilde{\eta} H_{1}^{\mathrm{i}}+\cdots\right) \\
\sigma & \sim \tilde{\eta}^{-1}\left(\sigma_{0}^{\mathrm{v}}+\tilde{\eta} \sigma_{1}^{\mathrm{v}}+\cdots\right) .
\end{aligned}
$$

Upon substituting these series expansions into the equations and boundary conditions, one obtains a sequence of second-order boundary value problems. In particular, for the sinuous eigenmode, it is found that

$$
H_{0}^{\mathrm{i}}=H_{0}^{\mathrm{o}}
$$

and

$$
\begin{aligned}
\sigma_{0}^{\mathrm{s}}= & -\left(m^{2}+k^{2}\right)\left(m^{2}+k^{2}-1\right) \\
\sigma_{1}^{\mathrm{s}}= & \frac{1}{9\left(m^{2}+k^{2}-1\right)}\left[9 \left(-k^{2}+k^{4}-2 m^{2}+5 k^{2} m^{2}-2 m^{2} k^{4}+4 m^{4}\right.\right. \\
& \left.\left.-4 k^{2} m^{4}-2 m^{6}\right)+4 k^{2} \beta\left(-6 k^{2}+k^{4}-6 m^{2}+7 k^{2} m^{2}+6 m^{4}\right)\right] .
\end{aligned}
$$

For the varicose mode, it is found that

$$
H_{0}^{\mathrm{i}}=-H_{0}^{\mathrm{o}}
$$




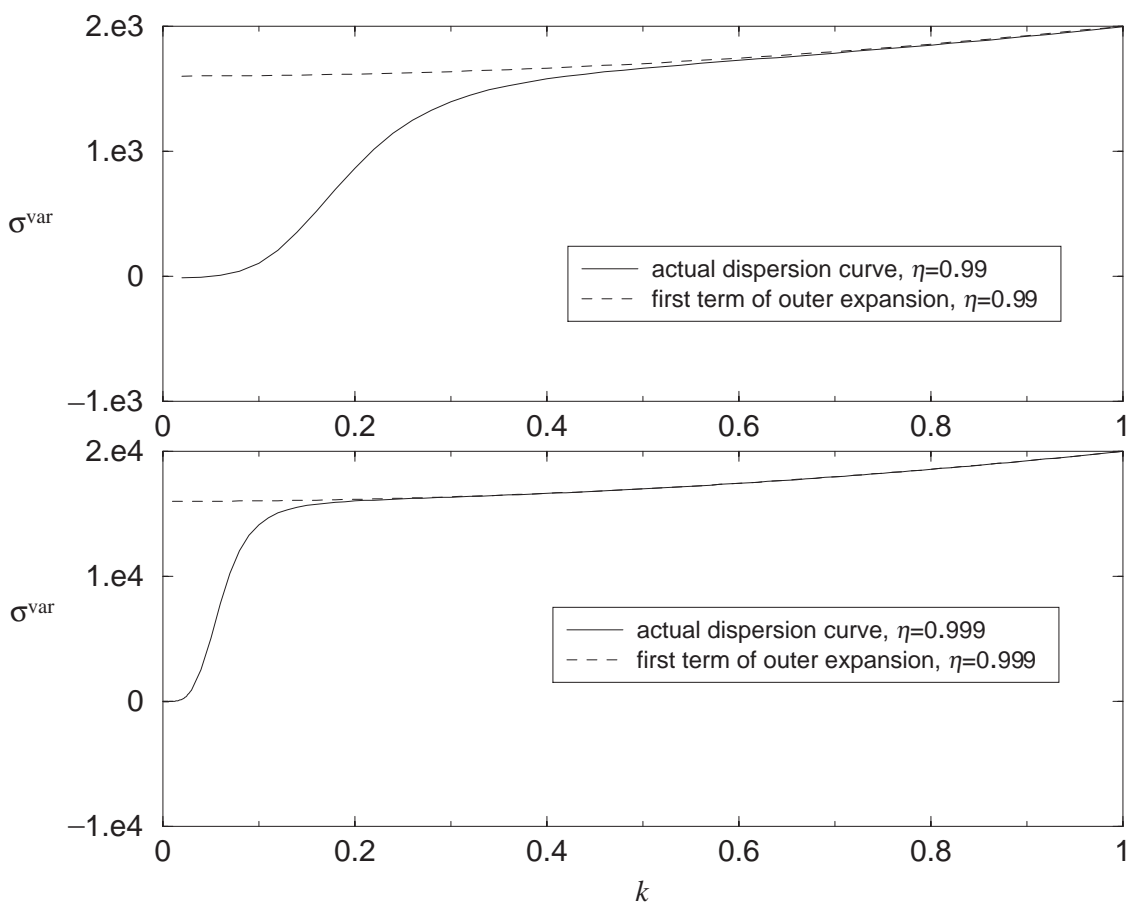

FIG. 19. Plots of varicose growth rate $\sigma^{\mathrm{var}}$ versus wavenumber $k$ for $m=2, \beta=1$ and $\tilde{\eta}=0.01$ (top) and 0.001 (bottom). Also plotted is the first term of the outer expansion, see equation (48). Notice the vertical scale in the two graphs.

$$
\sigma_{0}^{\mathrm{v}}=4 \beta\left(m^{2}+k^{2}\right)
$$

Some observations can be made at this point. First of all, from equations (44) and (47) the surface amplitudes are equal (at leading order). This is reasonable if one considers the geometry of a shell so thin that there is symmetry between the inner and outer surfaces (also, recall Fig. 8). Also, it appears that the sinuous growth rate is due to pure capillary effects at leading order (with elastic contributions entering at higher order), as though the two surfaces were acting as one perturbed surface with no material in between. On the other hand, the varicose growth rate is characterized by elastic effects at leading order (note the $\beta$ ), and is inversely proportional to $\tilde{\eta}$.

The leading-order varicose growth rate (48) is non-uniform in $k$ in the neighborhood of $k=0$, as can be seen by considering those modes with azimuthal wavenumber $m \geqslant 2$. These modes should have negative growth rates as $k \rightarrow 0$, consistent with the effects of capillarity. Figure 19 is a graph of the dispersion curve for $m=2, \beta=1$, and for $\tilde{\eta}=0.01$ and 0.001 . The boundary layers at $k=0$ are clearly visible. In addition, $\sigma$ is non-uniform in $k$ as $k \rightarrow \infty$ (capillary effects should have a dampening effect at large wavenumber). These non-uniformities in $k$ can be treated by seeking boundary layer solutions and finding a uniformly valid composite expansion.

4.7.2 Comparison with lamellar results of Sridhar et al. Consider again the thin-shell limit. On a length scale comparable to the shell thickness, the inner and outer radii are growing very large, and locally the shell resembles a flat plate. Hence, it is reasonable to compare the annulus 


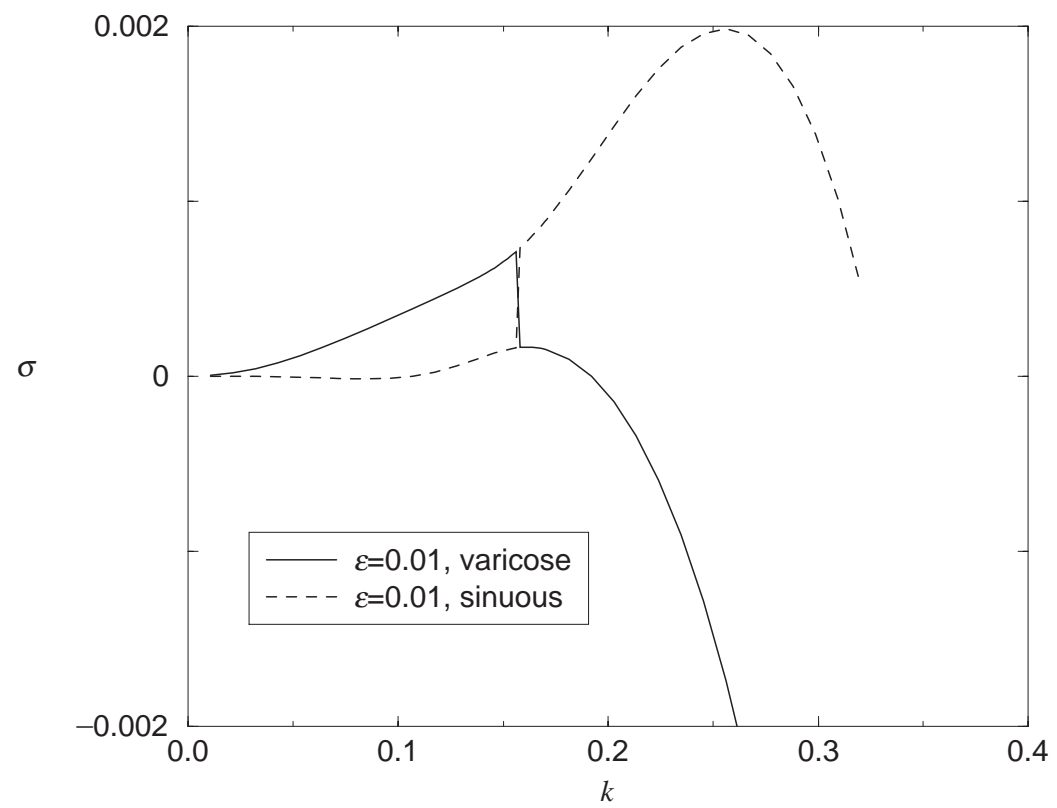

FIG. 20. Superposition of varicose and sinuous dispersion curves. Here, $\beta=0.011, m=0$, and $\epsilon=0.01$. Notice the discontinuity and growth rate switching which occurs between the two eigenmodes.

results to results dealing with morphological stability of lamellar microstructures. In particular, Sridhar et al. [16] examined the stability of lamellar eutectics, and plotted several graphs displaying maximal growth wavenumber versus elastic parameter. More specifically, they examined the stability of a uniaxially stressed plate-matrix composite, incorporating a plane-strain assumption. The top and bottom surfaces of their plate are comparable to our outer and inner surfaces, respectively, in the thin-shell limit.

In order to do a direct comparison, it is necessary to rescale variables using the thin-shell thickness $d \equiv R_{\mathrm{O}}-R_{\mathrm{i}}$, rather than the outer radius $R_{\mathrm{O}}$ as was done before. Therefore, $\beta$ becomes $\beta=\frac{d E \tilde{\varepsilon}^{2}}{2 \gamma}$, and a small parameter $\epsilon$ emerges, defined by $\epsilon \equiv \frac{d}{R_{0}}$. One should imagine $R_{\mathrm{o}}$ (and hence $R_{\mathrm{i}}$ ) growing large with thickness $d$ held fixed; hence, $\epsilon=\tilde{\eta}$.

According to [16], a maximal growth wavenumber of $k_{\max } \approx 0.16$ is obtained when parameter $\beta \approx 0.011$. It should be noted that Sridhar $e$ t al. obtained these numerical values by perturbing the top and bottom surfaces of the plate in a symmetrical fashion as viewed from the mid-plane of the plate. This would be analogous to a varicose perturbation of the annulus. In Fig. 20, the growth rate $\sigma$ is plotted against wavenumber $k$ (with $m=0$ ) for $\epsilon=0.01$. We see that the maximum varicose growth rate occurs approximately at $k=0.16$, hence the comparison to Sridhar et al. [16] is very good.

The thin-shell annulus limit compares favorably to their results in yet another way. Upon perturbing the plate in an anti-symmetric fashion, they found that the most dangerous mode is always sinuous rather than varicose (for any value of $\beta$ ). Examination of Fig. 20 tends to corroborate this finding. 


\section{Conclusions}

For the solid whisker with surface energy and surface diffusion [9], elastic-strain energy has a significant destabilizing influence, and can lead to the growth of nonaxisymmetric modes, in contrast to the single unstable axisymmetric mode when only surface tension effects are present. Furthermore, helical $(m=1)$ instabilities are preferred over a large range of elastic parameter $\beta$.

A different result is found for the pore (cylindrical hole case); the most dangerous mode (given by linear theory) is always axisymmetric. An increasingly greater range of azimuthal wavenumbers is excited as the applied stress is increased. In addition, modes of small axial wavenumber with azimuthal wavenumber $m=1$ are neutral in the case of the pore, but have positive growth rate in the case of the whisker.

The linear stability analysis of the tubule shows that as $\eta$, the ratio of inner radius to outer radius, approaches zero, the dispersion curves for one of the modes begin to resemble those of the solid whisker, and the curves for the other mode begin to resemble those of the pore. Furthermore, the 'solid-like' perturbation mode ('S') tended to concentrate near the outer surface, whereas the 'void-like' mode ('V') tended to concentrate near the inner surface. It is as if the tubule problem degenerates into the original solid whisker and pore problems as $\eta \rightarrow 0$. Further, the growth rate of the most dangerous mode (which is axisymmetric) is $O\left(\beta^{4}\right)$, and the corresponding wavenumber is $O(\beta)$, as $\beta \rightarrow \infty$, so that $\sigma_{\max } \sim 18 \beta^{4}$, and $k_{\max } \sim 2.7 \beta$, as $\beta \rightarrow \infty$ for any choice of $\zeta$ and $m$. Thus, large applied stresses compensate for differences in annular thicknesses and azimuthal wavenumbers. Also, as $k \rightarrow \infty$, the modes decouple, due to the rapid decay of the elastic field from the surfaces.

Finally, the phase relationship between inner and outer surface perturbations for the dominant mode can be either 'sinuous' or 'varicose' depending on the value of $\beta$. Indeed, for small values of applied stress the most dangerous mode is of varicose type, whereas values of $\beta$ larger than a critical value yields a sinuous disturbance. This result is somewhat counter-intuitive: a varicose perturbation with its periodic swelling would seem to store more elastic-strain energy (and hence provide a larger driving force for diffusion) than would a sinuous disturbance. However, results of Sridhar et al. [16] on varicose and sinuous perturbations of lamellar composites tend to corroborate these findings.

\section{Acknowledgements}

This work was supported in part by DOE grant DE-FG02-95ER25241 and NSF Nanoscale Interdisciplary Research Team grant DMR-0102794. DJK was supported by a Nicholson Fellowship while at Northwestern University.

\section{REFERENCES}

1. American Society for Metals. ASM Metals Reference Book, 2nd edn. Metals Park. ASM, OH (1983).

2. Asaro, R. J. \& Tiller, W. A. Interface morphology development during stress corrosion cracking: Part 1. Via surface diffusion. Met. Trans. 3, (1972) 1789-1796.

3. Bender, C. M. \& OrszaG, S. A. Advanced Mathematical Methods for Scientists and Engineers. McGraw-Hill, New York (1978).

4. Blakely, J. M. Introduction to the Properties of Crystal Surfaces. Pergamon, Oxford (1973).

5. Colin, J., GRIlhÉ, J., \& JunQUA, N. Morphological instabilities of a stressed pore channel. Acta Materialia 45, (1997a) 3835-3841. 
6. Colin, J., Grilhé, J., \& JunQua, N. Surface instabilities of a stressed cylindrical whisker. Phil. Mag. 76, (1997b) 793-805.

7. GRINFEL'D, M. A. Instability of the separation boundary between a non-hydrostatically stressed elastic body and a melt. Sov. Phys. Doklady 31, (1986) 831-834.

8. KIRILL, D. J. Morphological instability of whiskers, pores and tubules, Ph.D. Thesis, Northwestern University, (1999).

9. Kirill, D. J., Davis, S. H., Miksis, M. J., \& Voorhees, P. W. Morphological instability of a whisker. Proc. R. Soc. Lond. A 455, (1999) 3825-3844.

10. McCallum, M. S., Voorhees, P. W., Miksis, M. J., Davis, S. H., \& Wong, H. Capillary instabilities in solid thin films: lines. J. Appl. Phys. 79, (1996) 7604-7611.

11. Mullins, W. W. Theory of thermal grooving. J. Appl. Phys. 28, (1957) 333-339.

12. Nichols, F. A. \& Mullins, W. W. Surface- (interface-) and volume-diffusion contributions to morphological changes driven by capillarity. Trans. Metal. Soc. AIME 233, (1965) 1840-1848.

13. Powers, J. D. \& Glaeser, A. M. High-temperature healing of cracklike flaws in titanium ionimplanted sapphire. J. Amer. Ceramic Soc. 76, (1993) 2225-2234.

14. Sokolnikoff, I. S. Mathematical Theory of Elasticity. McGraw-Hill, New York (1956).

15. Spencer, B. J., Voorhees, P. W., \& Davis, S. H. Morphological instability in epitaxially strained dislocation-free solid films: linear stability theory. J. Appl. Phys. 73, (1993) 4955-4970.

16. SRIDHAR, N., Rickman, J. M., \& SROLOVITZ, D. J. Microstructural stability of stressed lamellar and fiber composites. Acta Materialia 45, (1997) 2715-2733.

17. Srolovitz, D. J. \& SAfran, S. A. Capillary instabilities in thin films. II. Kinetics. J. Appl. Phys. 60, (1986) 255-260.

18. Srolovitz, D. J. \& Thompson, C. V. Beading instabilities in thin film lines with bamboo microstructures. Thin Solid Films 139, (1986) 133-141.

19. Wolfram, S. Mathematica: A System for Doing Mathematics by Computer, 2nd edn. Addison Wesley, Redwood City, CA (1991). 